\title{
Boundary Field Theory Approach to the Renormalization of SQUID Devices
}

\author{
Domenico Giuliano ${ }^{1}$ and Pasquale Sodano ${ }^{2}$ \\ ${ }^{1}$ Dipartimento di Fisica, Università della Calabria and I.N.F.N., Gruppo collegato \\ di Cosenza \\ Arcavacata di Rende I-87036, Cosenza, Italy \\ 2 Progetto Lagrange- Fondazione C.R.T. and Fondazione I.S.I., \\ Dipartimento di Fisica, Politecnico di Torino, \\ Corso Duca degli Abruzzi 24, Torino, Italy ${ }^{1}$
}

\begin{abstract}
We show that the quantum properties of some Josephson SQUID devices are described by a boundary sine Gordon model. Our approach naturally describes multi-junction SQUID devices and, when applied to a single junction SQUID (the rf-SQUID), it reproduces the known results of Glazman and Hekking. We provide a detailed analysis of the regimes accessible to an rf-SQUID and to a two-Josephson junction SQUID device (the dc-SQUID). We then compute the normal component of the current-response of a SQUID device to an externally applied voltage and show that the equation describing the current-voltage characteristic function reduces to well-known results when the infrared cutoff is suitably chosen. Our approach helps in establishing new and interesting connections between superconducting devices, quantum brownian motion, fermionic quantum wires and, more generally, quantum impurity problems.
\end{abstract}

Key words: Boundary conformal field theories, Superconducting quantum interference devices (SQUIDs)

PACS: 03.70.+k , 11.25.Hf , 85.25.Dq, 85.25.Cp

\section{Introduction}

Quantum effects in Josephson junctions have been by now investigated for quite a long time, both experimentally [1] and theoretically [2]: the most

1 Permanent address: Dipartimento di Fisica, Università di Perugia, and I.N.F.N., Sezione di Perugia, Via A. Pascoli, 06123, Perugia, Italy 
important manifestation of quantum fluctuations in these systems being the macroscopic quantum tunneling of the phase across a current biased junction and the consequent observation of quantum phase slips events at a bias rather close to the Josephson critical current [1].

Early studies of quantum phase fluctuations in Josephson systems have been carried using either the Caldeira- Leggett [3] or the electromagnetic environment [4] models: within this framework, it has been shown that macroscopic quantum tunneling causes a finite voltage to appear for any finite current, leading to a non-linear I-V characteristic which, in the limit of zero temperature, reads as $V=A I^{\gamma}$ with $\gamma$ depending on the impedance of the superconducting leads and $A$ being proportional to the bare tunnel matrix element between two adjacent minima of the potential [5]. The dual result for a voltage biased junction shows that the dc-current in such a junction is proportional to the square of the unrenormalized Josephson energy [6].

As already pointed out in Ref.[7], in the above mentioned approaches the effective boundary conditions for the quantum fluctuations of the "environment modes" do not depend on the Josephson energy of the junction. While this assumption is perfectly legitimate for weak fluctuations of the phase of the order parameter across the junction, better care should be used if these fluctuations are strong as it may well happen for one-dimensional superconductors, where phase fluctuations diverge logarithmically with the length of the system, opening the undesirable option that the Josephson energy could average to zero for diverging random fluctuations of the phase.

In their seminal paper Glazman and Hekking [7] showed that a finite renormalized Josephson energy may arise since the junction itself affects the fluctuations of the environment. They considered an rf-SQUID, i.e. a thin superconducting loop containing a Josephson junction, phase-biased by threading a flux $\Phi_{\text {ext }}$ through the loop. The environment modes of the loop consist of plasmon modes propagating through a superconducting wire with a soundlike dispersion law [8]. Their analysis was based on a perturbative renormalization group approach exploiting the similarity of the effective action, emerging from integrating out the fluctuations "away" from the junction, with the one used to analyze the quantum brownian motion in a periodic potential [9]. They found how the Josephson coupling $E_{J}$ is renormalized when high energy degrees of freedom are integrated out and showed that the renormalization leads to values of the Josephson critical current which are smaller than one would expect from the mean field theory result $J_{c}^{0}=\pi \Delta G / 2 e$, where $G$ is the conductance of the junction and $\Delta$ is the superconducting gap in the loop. The $\mathrm{RG}$ approach is supplemented by an instanton analysis [10] needed to compute the effect of the macroscopic quantum tunneling on the phase dependence of the Josephson current for relatively large loops. 
Generalized SQUID devices are much studied today mainly because of their relevance for the implementation of flux qu-bits [11]: it is by now widely accepted that multi-junction SQUID devices [12] are the most promising realization of a flux qu-bit due to their reduced size, which renders them less sensitive to decoherence effects [13] induced by the interaction with the environment. As it is well known, an rf-SQUIDs [14 may be represented as a two level system only when the superconducting loop is rather large and, thus, unfortunately more sensitive to interactions with the environment.

In this paper we evidence that all the non-perturbative renormalization effects induced by quantum fluctuations of the phase of the superconducting order parameter [7] are described by the two-boundary sine Gordon model [15], which becomes then the pertinent description of the dynamics of the fluctuation field and of a Josephson junction with arbitrary nominal strength $E_{J}$. As we shall see, the quantum behaviors of rf-SQUIDs and of multi-junction SQUID devices, may be analyzed within the framework of boundary field theories [16].

A two boundary sine Gordon model description of SQUID devices is possible since the dynamics of the fluctuations of the phase of the superconducting wire may be described as a spinless Tomonaga-Luttinger liquid (TLL) [17]. In fact, for a superconducting wire of length $2 L$ and cross-sectional area $S$, the Lagrangian describing the phase fluctuations of the order parameter may be written as [7]:

$$
L=\int_{-L}^{L} d x\left\{\frac{\hbar^{2}}{2 e_{c}}\left(\frac{\partial \Phi}{\partial t}\right)^{2}-\frac{\hbar^{2} n_{s} S}{4 m}\left(\frac{\partial \Phi}{\partial x}\right)^{2}\right\}
$$

where $n_{s}$ is the superfluid density, $m$ is the electron mass, $1 / e_{c}$ is the characteristic inverse charging energy per unit length of the loop:

$$
\frac{1}{e_{c}}=\frac{\epsilon}{8 e^{2} \ln (R / a)}
$$

where $\epsilon$ is the dielectric constant of the medium surrounding the loop, while $R$ is the distance from a metallic screen, which, together with $a$, fixes the lead's capacitance.

Upon setting $\hbar=1$, if one defines the "Luttinger" parameter $g=\frac{\pi}{2} \sqrt{n_{s} S /\left(m e_{c}\right)}$ and the plasmon velocity $v_{\mathrm{pl}}=\sqrt{n_{s} S e_{c} /(4 m)}$, one immediately sees that the Lagrangian (1) may be written as the one describing a spinless Luttinger liquid 
[18]:

$$
L=\frac{g}{2 \pi} \int_{-L}^{L} d x\left\{\frac{1}{v_{\mathrm{pl}}}\left(\frac{\partial \Phi}{\partial t}\right)^{2}-v_{\mathrm{pl}}\left(\frac{\partial \Phi}{\partial x}\right)^{2}\right\} .
$$

Physically, $1 / \mathrm{g}$ is the dimensionless zero-frequency impedance of the superconducting wire [7,20]. Due to its dependence on $S, g$ may take values in a rather wide interval of real numbers: for $g>1$, Eq.(3) describes a TLL with an attractive interaction, while, for $g<1$ the interaction is repulsive. The value $g=1$ describes the very special case of an essentially free theory. Different superconducting wires may realize different values of $g$ : for instance, for rather clean aluminum wires, one can easily attain values of $g$ such that $g>1$ and, thus, realize a wire for which the dynamics of the phase fluctuations is described by an attractive TLL.[7].

For $g>1$, the two boundary sine Gordon model description of a SQUID device allows to make very general statements regarding the regimes accessible to the system for a finite loop-size $L$. Namely, there will be a perturbative (in $E_{J}$ ) weak coupling regime accessible when the relevant Josephson couplings are small and a non-perturbative strong coupling regime accessible when $E_{J}$ becomes large; most importantly, there will be a renormalization group invariant length scale $L_{*}$ such that for $L<L_{*}$ the SQUID device is in the perturbative weak coupling regime, while it is in the non-perturbative strong coupling regime for $L>L_{*}$. Intuitively speaking, $L_{*}$ is (2 $2 \pi$ times) the radius for which the ratio between the Josephson energy and the magnetic energy due to the loop self-inductance equals one; the existence of an "healing length" $L_{*}$ is generic to a boundary sine Gordon model describing a TLL [21]. At variance, for $g<1$, the theory lies within the perturbative regime at any $L$. Therefore, there is no healing length in this case and no universal behavior is then attainable. In the following, we shall show that these properties are shared by a variety of SQUID devices.

We investigate also the stability of the two (weakly and strongly coupled) regimes by looking at the relevance/irrelevance of the operators describing the boundary interactions. It turns out that these operators are either vertex operators or instantons (phase slips) of the plasmon field $\Phi$, depending on if the system is within the weakly, or the strongly coupled regime. As evidenced in the following, vertex (instantons) are a relevant (irrelevant) perturbation

2 Our normalization of $g$ renders the boundary interaction marginal when $g=1$. It differs by the one used in Ref.[19] by a factor of $1 / 2$. 
of the weakly (strongly) coupled regime for $g>1$, while they are irrelevant (relevant) for $g<1$.

It emerges the picture that, for $g>1$, it always exists an healing length $L_{*}$ such that, for $L>L_{*}$, the Josephson coupling affects the dynamics of the plasmon field as a boundary condition: in this regime it is impossible to disentagle the Josephson junction from the environmental modes. For $g<1$, instead, this situation is never attainable and the Josephson coupling has to be always regarded as a quantum impurity embedded in a superconducting wire.

The strongly and weakly coupled regimes may be probed by looking either at the dc-conductance, or at the behavior of the Josephson current as a function of the magnetic flux treading the superconducting loop. The two-boundary sine Gordon approach provides a systematic and powerful method to evaluate the relevant currents. In fact, the correlators of the plasmon fluctuation $\Phi$ field(s) may be exactly computed and this allows for a systematic derivation of the leading corrections to the Josephson current for all values of the applied flux $\varphi=\Phi_{\text {ext }} / \Phi_{0}^{*}\left(\Phi_{0}^{*}=h /(2 e)\right)$, which, for $\varphi=\pi$, yields the results of the instanton analysis carried in Ref.[7]; in addition, we shall show that it also allows for a systematic derivation of the response of a SQUID device to an applied external voltage. Since applying a finite voltage $V$ introduces a new energy scale $e^{*} V$, with $e^{*}=2 e$, the dc-current response of the system strongly depends on the ratio between the new energy scale, and the intrinsic scale provided by the level spacing $s=2 \pi v_{\mathrm{pl}} / L$ of the plasmon field(s) $\Phi$. Our computation of the dc-current clearly shows that, when $e^{*} V>s$, one gets the well known Kane-Fisher formula [9], while, for $s>e^{*} V$, one gets the results obtained by Glazman and Hekking [7]; in addition, when $e^{*} V>s$, the dc-current dominates over the Josephson current.

Boundary field theories have by now become relevant in several different contexts. In condensed matter theory, they are mostly generalizations of quantum impurity models, which may be described by using the TLL paradigm [17]; for instance, boundary interactions appear in the analysis of the Kondo problem [22], in the study of a one-dimensional conductor in presence of an impurity [23], in the derivation of tunneling between edge states of a Hall bar [24], in the study of quantum brownian motion in a periodic potential [25] and in the analysis of the phases accessible to networks of quantum wires [19] and Josephson junctions [26],[21]. The TLL paradigm evidences that many interactions are simply diagonalizable in the basis of pertinent collective bosonic modes, and that non-diagonalizable interactions correspond to exactly solvable Hamiltonians, such as sine Gordon models [27]. Recently, boundary field theories have been investigated in the context of string theories. For instance, in studying tachyon instabilities [28], one is faced with the fact that the space of interacting string theory [29] is mapped onto the space of boundary per- 
turbations of conformal field theories [30], and that the renormalization group flow determined by boundary perturbations may be identified with tachyon condensation [31]. Affleck and Ludwig [32] showed that the boundary entropy $g$ is decreasing along the renormalization group trajectories, triggered by the boundary interactions. It is remarkable that this entropy has been proposed as a measure of entanglement for boundary states [32].

The paper is organized as follows:

Section 2 shows that quantum behaviors of an rf-SQUID are described by a two-boundary sine Gordon model. There, we shall analyze the weak and the strong coupling regime accessible to the device for a finite size of the superconducting loop. We show that, for $g>1$, there is a renormalization group invariant scale - the "healing length" $L^{*}$ - [7] marking the crossover between the weakly coupled and the strongly coupled regime.

We first analyze the weakly coupled fixed point corresponding to mixed boundary conditions (i.e. Dirichlet at the inner boundary and Neumann at the outer boundary) and we determine the scale dimension of the pertinent boundary operators and the scaling equations for the running coupling constants using renormalization group ( $R G$ ) methods; this allows for a perturbative computation of the beta-function for all the couplings. Computation of the canonical ground state energy shows that- at the weakly coupled fixed point- the Josephson current has a sine dependence on the flux treading the loop. Then, we analyze the strong coupling fixed point, corresponding to Dirichlet boundary conditions at the outer and inner boundaries of the rf-SQUID. Evaluation of the canonical ground state energy shows that- at the strongly coupled Dirichlet fixed point- the Josephson current exhibits a saw-tooth dependence on the external flux.

Due to the zero modes, appearing in the strong coupling regime as a consequence of the Dirichlet boundary conditions, the ground state is doubly degenerate when $\varphi=\pi+2 \pi k$ : when these levels are confined away from the rest of the spectrum, the rf-SQUID realizes a qu-bit (See, for instance, Ref.[11]). Our analysis points out that this realization is possible only for $g>1$ and for sizes of the superconducting loop bigger than the healing length, and, thus, for a rather big self-inductance of the device. Finally, we analyze the effects of quantum phase slips on the ground state degeneracy of the rf-SQUID at the strong coupling fixed point: an explicit instanton computation shows that, for $g>1$, the degeneracy is lifted by an amount proportional to the instanton fugacity, and that the instantons smoothen the edges of the saw-tooth phase-current relation.

In section 3 we analyze a SQUID-device involving only two Josephson junctions, of arbitrary strength $E_{J, 1}, E_{J, 2}$ (the dc-SQUID). Under the assumption 
that the two plasmon fields living in the two branches of the superconducting loop (see fig.1) are described by TLLs corresponding to the same $g$, we show that also the dc-SQUID is described by a two-fields generalization of the two boundary sine Gordon model. We show that also here, for $g>1$, there is an unstable weak coupling fixed point and a stable one at strong coupling and that the crossover from one to the other is characterized by an healing length $L^{*}$, whose value depends, of course, only on the smallest of the two Josephson couplings. When $g<1$, the only stable fixed point is at weak coupling.

In section 4 we analyze the normal component of the current-response of the rf- and dc-SQUIDs to an applied external voltage. There, we evidence that a computation of the time-independent current using the $\Phi$-field(s) correlators derived from the two-boundary sine Gordon model yields the well-known Kane-Fisher formula when the infrared cutoff $e^{*} V>s$ [9], as well as the Glazman-Hekking result [7], when the infrared cutoff if $e^{*} V<s$. It is remarkable that, as a result of the boundary field theory approach to superconducting SQUID devices developed in this paper, one gets a current-voltage equation valid for any choice of the infrared cutoff. Furthermore, for a dc-SQUID, the boundary field theory approach evidences the possibility of new remarkable interference effects (even in the absence of an external applied magnetic flux (!)) between the currents flowing across the two junctions.

Section 5 is devoted to our conclusions and final remarks.

In order to be pedagogical and self-contained, a few pertinent mathematical details are summarized in the appendices.

\section{The rf-SQUID}

From Eq.(3) one may easily obtain the Hamiltonian $H_{r f}$ for an rf-SQUID (i.e. for a superconducting wire cut by a Josephson junction with nominal Josephson coupling $E_{J}$ and pierced by a magnetic flux $\varphi$ ). By neglecting the charging energy of the junction [7], $H_{r f}$ is given by:

$$
\begin{aligned}
& H_{r f} \equiv H_{0}+H_{B}=\frac{g}{2 \pi} \int_{-L}^{L} d x\left[\frac{1}{v_{\mathrm{pl}}}\left(\frac{\partial \Phi(x)}{\partial t}\right)^{2}+v_{\mathrm{pl}}\left(\frac{\partial \Phi(x)}{\partial x}-\frac{\varphi}{2 L}\right)^{2}\right] \\
& -E_{J} \cos [\Phi(L)-\Phi(-L)],
\end{aligned}
$$

where $H_{0}$ and $H_{B}$ describe the bulk and boundary Hamiltonian, respectively.

The flux $\varphi$ may be accounted for by a redefinition of the field $\Phi$ as $\Phi \rightarrow$ $\Phi-\varphi \frac{x}{2 L}$. By introducing the symmetric and the antisymmetric phase fields 
$\Phi_{e}(x)=\frac{1}{\sqrt{2}}[\Phi(x)+\Phi(-x)]$ and $\Phi_{o}(x)=\frac{1}{\sqrt{2}}[\Phi(x)-\Phi(-x)]$, with $0 \leq x \leq L$, one gets

$$
H_{r f}=\sum_{j=e, o} \frac{g}{2 \pi} \int_{0}^{L} d x\left[\frac{1}{v_{\mathrm{pl}}}\left(\frac{\partial \Phi_{j}(x)}{\partial t}\right)^{2}+v_{\mathrm{pl}}\left(\frac{\partial \Phi_{j}(x)}{\partial x}\right)^{2}\right]-E_{J} \cos \left[\sqrt{2} \Phi_{o}(L)+\varphi\right]
$$

The field $\Phi_{e}(x)$ fully decouples from the interaction term in Eq.(15). The Hamiltonian for the field $\Phi_{o}(x)$ (which will be referred to as $\Phi(x)$, from now on), on the other hand, is the Hamiltonian for a two-boundary sine Gordon model [15], with Dirichlet boundary condition at the inner boundary, i.e., $\Phi(0, t)=0$, and dynamical boundary conditions at the outer boundary. The latter are obtained by requiring that the energy functional is conserved, yielding [21]

$$
\frac{g v_{\mathrm{pl}}}{\pi} \frac{\partial \Phi(L, t)}{\partial x}+\sqrt{2} E_{J} \sin [\sqrt{2} \Phi(L, t)+\varphi]=0 .
$$

For $E_{J} \rightarrow 0$, one has Neumann boundary conditions at the inner boundary, i.e., $\frac{\partial \Phi(L, t)}{\partial x}=0$, while, for $E_{J} \rightarrow \infty$, one gets Dirichlet boundary conditions, $\sqrt{2} \Phi(L, t)=-\varphi+2 \pi k, k \in Z$. As it will be clearer in the following, the effective two-boundary sine Gordon model provides the relevant renormalizations

of an rf-SQUID: namely, it gives the renormalized value $\left(\bar{E}_{J}\right)$ of the Josephson energy and yields the correct functional dependence of the Josephson current, as a function of the flux $\varphi$.

As we shall see in the following sections, as $L \rightarrow \infty$, there are two relevant fixed points, namely, the weakly coupled Neumann fixed point $\left(E_{J}=0\right)$, and the strongly coupled Dirichlet fixed point $\left(E_{J}=\infty\right)$. Our analysis will evidence the existence of an healing length $L_{*}=g \pi v_{\mathrm{pl}} / E_{J}$, providing the size of a loop for which the ratio between the Josephson energy and the magnetic energy due to the loop self-inductance equals 1 , such that, for $g>1$, at $L=L_{*}$ the device crosses over from the weakly to the strongly coupled regime.

\subsection{The weakly coupled fixed point}

At the weakly coupled fixed point $\left(E_{J} \rightarrow 0\right)$, the field $\Phi(x, t)$ obeys Dirichlet boundary conditions at the inner boundary and Neumann boundary conditions at the outer boundary. As a consequence, $\Phi(x, t)$ takes the mode expansion

$$
\Phi(x, t)=-\sqrt{\frac{1}{g}} \sum_{n \neq 0} \frac{\alpha(n)}{n} \sin \left(k_{n} x\right) e^{-i k_{n} u t}
$$


with $\left[\alpha(n), \alpha\left(n^{\prime}\right)\right]=n \delta_{n+n^{\prime}, 0}$, and $k_{n}=\frac{\pi}{L}\left(n+\frac{1}{2}\right)$.

The ground state of the bosonic theory, $|\mathrm{GS}\rangle$, is defined in terms of the oscillatory modes as

$$
\alpha(n)|\mathrm{GS}\rangle=0 \quad ; \forall>0
$$

Normal ordering the Josephson energy with respect to $|\mathrm{GS}\rangle$, leads to

$$
H_{J}=-\frac{E_{J}}{2}\left(\frac{2 \pi a}{L}\right)^{\frac{1}{g}}\left[: e^{i \sqrt{2} \Phi(L)+i \varphi}:+: e^{-i \sqrt{2} \Phi(L)-i \varphi}:\right]
$$

where the column :: denotes, as usual, normal ordering.

The thermodynamic limit (i.e., large $L$ ) is attained once the scaling equations for the pertinent running couplings have been obtained using the renormalization group (RG) approach (see appendix A).

The RG equations are determined by the requirement that the partition function $\mathbf{Z}$ is independent of the cutoff. In order to compute $\mathbf{Z}$, one should Wickrotate the field $\Phi$ and resort to the imaginary-time Feynman path-integral formalism. The partition function is, then, given by

$$
\mathbf{Z}=\int \mathbf{D} \Phi e^{-S_{0}} \mathbf{T}_{\tau}\left[e^{-S_{B}}\right]
$$

where $\mathbf{T}_{\tau}$ is the imaginary time ordering operator, $S_{0}$ is the free Euclidean action

$$
S_{0}=\frac{g}{2 \pi} \int_{0}^{\beta} d \tau \int_{0}^{L} d x\left[\frac{1}{v_{\mathrm{pl}}}\left(\frac{\partial \Phi}{\partial \tau}\right)^{2}+v_{\mathrm{pl}}\left(\frac{\partial \Phi}{\partial x}\right)^{2}\right]
$$

and $S_{B}$ is the boundary interaction action at imaginary times

$$
S_{B}=-\frac{\bar{E}_{J}}{2} \int_{0}^{\beta} d \tau\left[: e^{i[\sqrt{2} \Phi(i \tau)+\varphi]}:+: e^{-i[\sqrt{2} \Phi(i \tau)+\varphi]}:\right]
$$

with $\Phi(i \tau)=\Phi(L, i \tau)$.

In Eq.(12), the renormalized coupling constant $\bar{E}_{J}=E_{J}(2 \pi a / L)^{\frac{1}{g}}$ has been introduced. Of course, after this redefinition of the Josephson coupling, there is not anymore a cutoff dependence of the interaction operator [33]. 
The boundary action in Eq.(12) contains only the vertex operators $V_{ \pm 1}(\tau)=$ $e^{ \pm i \varphi}: \exp [ \pm i \sqrt{2} \Phi(i \tau)]$ :. Due to the mixed boundary conditions on $\Phi$, the operator $\Phi(i \tau)$ contains no zero modes and, thus, there are no selection rules on the Kac-Moody charge carried by the vertex operators. As a consequence, the Operator Product Expansions (O.P.E.'s) may generate additional vertices, with higher periodicity in $\varphi$, given by

$$
V_{n}(\tau)=e^{ \pm n i \varphi}: \exp [ \pm i \sqrt{2} n \Phi(i \tau)]: \quad .
$$

The scaling dimension of $V_{n}, h_{n}$, is derived from the correlation function $\left\langle T_{\tau}\left[V_{n}(\tau) V_{-n}(\tau)\right]\right\rangle$ which, for $\beta \rightarrow \infty$, is approximatively given by (see Appendix $\mathrm{B})$

$$
\left\langle T_{\tau}\left[V_{n}(\tau) V_{-n}\left(\tau^{\prime}\right)\right]\right\rangle \approx\left|\tanh \left[\frac{\pi v_{\mathrm{pl}}}{4 L}\left(\tau-\tau^{\prime}\right)\right]\right|^{-\frac{2 n^{2}}{g}} .
$$

At short imaginary time distances $\left(v_{\mathrm{pl}}\left|\tau-\tau^{\prime}\right| / L \ll 1\right)$, Eq.(14) becomes

$$
\left\langle T_{\tau}\left[V_{n}(\tau) V_{-n}\left(\tau^{\prime}\right)\right]\right\rangle \approx\left|\frac{\pi v_{\mathrm{pl}}}{L}\right| \tau-\tau^{\prime}||^{-\frac{2 n^{2}}{g}}
$$

leading to $h_{n}=n^{2} / g$.

As we shall see in the following, despite the fact that $h_{n}$ depends on $n$, due to nonlinearities, the higher harmonics in Eq.(13) are irrelevant operators, when $g<1$, while they become relevant for $g>1$.

The O.P.E. yields the pertinent fusion rules for the vertex operators

$$
V_{n}(\tau) V_{n^{\prime}}\left(\tau^{\prime}\right) \approx_{\tau^{\prime} \rightarrow \tau}\left[\frac{\pi v_{\mathrm{pl}}}{L}\left(\tau-\tau^{\prime}\right)\right]^{-h_{n}-h_{n^{\prime}}+h_{n+n^{\prime}}} V_{n+n^{\prime}}\left(\tau^{\prime}\right)
$$

Introducing the pertinent counterterms, one may easily show that the effective boundary action looks as

$$
S_{B, E f f}=-\frac{1}{2} \int_{0}^{\beta} d \tau \sum_{n=1}^{\infty}\left\{\bar{E}_{n} e^{i n \varphi} V_{n}(\tau)+\bar{E}_{n} e^{-i n \varphi} V_{-n}(\tau)\right\}
$$


where the renormalization condition is that all the coupling strengths $\bar{E}_{n}=$ $(2 \pi a / L)^{\frac{n^{2}}{g}} E_{n}$, with $n \neq \pm 1$, vanish at a given reference length scale $L_{0}$.

Setting the running couplings $g_{n}$ as

$$
g_{n}=\left(\frac{L}{2 \pi a}\right)^{1-\frac{n^{2}}{g}} \frac{a^{n} E_{n}}{v_{\mathrm{pl}}^{n}}
$$

from the fusion rules given in Eq.(16), one is able to derive, in principle, the $\beta$-function for all the couplings $g_{n}$.

For the sake of simplicity, we shall restrict our attention to the first two relevant terms, proportional to the running couplings $g_{1}, g_{2}$. The RG equations are, then

$$
\frac{d g_{1}}{d \ln \left(L / L_{0}\right)}=\beta_{1}\left(g_{1}, g_{2}\right)=\left(1-\frac{1}{g}\right) g_{1}+g_{1} g_{2}
$$

and

$$
\frac{d g_{2}}{d \ln \left(a / a_{0}\right)}=\beta_{2}\left(g_{1}, g_{2}\right)=\left(1-\frac{4}{g}\right) g_{2}+\left(g_{1}\right)^{2} \quad:
$$

the flux $\varphi$ fully decouples from Eqs.(1920) and, thus, the scaling near the Neumann fixed point is independent of $\varphi$.

For $g<1, g_{1}$ and $g_{2}$ are both irrelevant. Thus, the Neumann fixed point is stable and the theory is perturbative in the boundary interaction with effective, size-dependent couplings given by

$$
g_{1}(L) \approx g_{1}\left(L_{0}\right)\left(\frac{L}{L_{0}}\right)^{\left(1-\frac{1}{g}\right)} \quad ; g_{2}(L) \approx 0
$$

On the other hand, when $g>1, g_{1}$ becomes relevant since it grows with $L$ as

$$
g_{1}(L) \approx g_{1}\left(L_{0}\right)\left(\frac{L}{L_{0}}\right)^{\left(1-\frac{1}{g}\right)}
$$


By inserting Eq.(22) into the nonlinear term of Eq.(20), one gets

$$
g_{2}(L) \approx \frac{\left(g_{1}^{2}(L)\right)}{1+2 / g}\left[1-\left(\frac{L}{L_{0}}\right)^{-1-\frac{2}{g}}\right],
$$

where the integration constants have been chosen so as to be consistent with the renormalization condition $g_{2}\left(L_{0}\right)=0$.

From Eqs.(23), one sees that the nonlinear term in Eq.(19) makes $g_{2}(L)$ increase, as soon as one starts the scaling flow, even when $1-4 / g<0$. This is a remarkable result, since it implies that, as soon as the first harmonics in $\varphi$ sets in, all the others follow, due to nonlinearities in the RG equations.

At the Neumann fixed point, the Josephson current is perturbative in $\bar{E}_{J}$ : of course, as $L$ increases, our results are reliable only for $g<1$. As a function of the flux $\varphi, I[\varphi]$, is given by

$$
I[\varphi]=\frac{1}{c \Phi_{0}^{*}} \lim _{\beta \rightarrow \infty} \frac{1}{\beta} \frac{\partial}{\partial \varphi} \ln \left[\frac{\mathbf{Z}_{E f f}[\varphi]}{\mathbf{Z}_{0}}\right],
$$

where $\mathbf{Z}_{E f f}[\varphi]$ is the $\varphi$-dependent partition function, given by

$$
\mathbf{Z}_{E f f}[\varphi]=\int \mathbf{D} \Phi e^{-S_{0}} \mathbf{T}_{\tau}\left[e^{-S_{B, E f f}}\right]
$$

To the second order in $g_{n}$, one gets

$$
I[\varphi]=e^{*} E_{J}\left(\frac{2 \pi a}{L}\right)^{\frac{1}{g}}\left[\sin (\varphi)+\frac{g_{2}(L)}{g_{1}(L)} \sin (2 \varphi)\right]
$$

which explicitly gives the contribution of the second harmonics to the Josephson current.

For $g>1$, Eq.(23) shows that, for $L \rightarrow \infty$, the ratio between the running coupling constants of the first two harmonics is given by

$$
\frac{g_{2}(L)}{g_{1}(L)} \propto\left(\frac{L}{L_{0}}\right)^{1-\frac{1}{g}}
$$

showing the relevance of the boundary interaction, since higher harmonics become more and more relevant, as $L \rightarrow \infty$. The scaling equations (18, 19,20,21) 
cease to be valid at the renormalized healing length $\bar{L}_{*}$ for which $g_{1}\left(\bar{L}_{*}\right) \sim 1$, i.e., when

$$
L=\bar{L}_{*}=2 \pi a\left(\frac{v_{\mathrm{pl}}}{a E_{J}}\right)^{\frac{g}{g-1}}
$$

yielding that the renormalized Josephson coupling $\bar{E}_{J}^{*}=\bar{E}_{J}\left(L_{*}\right)$ is given by

$$
\bar{E}_{J}^{*}=\left(\frac{a E_{J}}{v_{\mathrm{pl}}}\right)^{\frac{1}{g-1}} E_{J}
$$

which is the energy where the level spacing of the plasmon field becomes of the order of the Josephson energy, i.e., $2 \pi v_{\mathrm{pl}} / \bar{L}_{*} \sim\left(2 \pi a / \bar{L}_{*}\right)^{\frac{1}{g}} E_{J}[7]$.

\subsection{The strongly coupled fixed point}

In this subsection, we analyze the properties of the rf-SQUID in the limit in which $\bar{E}_{J} /\left(2 \pi v_{\mathrm{pl}} / L\right)$ is $\gg 1: H_{J}$ is now the dominating potential term, and the field $\Phi(L, t)$ takes values corresponding to a minimum of the boundary energy. Therefore, at both boundaries $\Phi(x)$ must obey to Dirichlet boundary conditions, given by

$$
\Phi(0, t)=0 ; \sqrt{2} \Phi(L, t)=-\varphi+2 \pi k ;(k \in Z)
$$

Both boundary conditions are satisfied if one chooses for $\Phi(x, t)$ the mode expansion

$$
\Phi(x, t)=-\frac{2 \pi x}{L} P-\sqrt{\frac{1}{g}} \sum_{n \neq 0} \frac{\alpha(n)}{n} \sin \left(k_{n} x\right) e^{i k_{n} v_{\mathrm{pl}} t}
$$

where $P$ is the zero mode operator and $k_{n}=\left(\frac{\pi}{L} n\right)$. From Eq.(30), one obtains the set of possible eigenvalues of $P$ :

$$
P_{k}=\frac{1}{\sqrt{2}}\left[-\frac{\varphi}{2 \pi}+k\right] \text {. }
$$

As a consequence, there will be an infinite set of ground states of the oscillatory modes, corresponding to the possible eigenvalues of $P$, and denoted by $\left|P_{k},\{0\}\right\rangle$. 
Once $H_{r f}$ has been evaluated for the particular solution given in Eq.(31), one obtains the effective Hamiltonian at the Dirichlet fixed point, $H_{D}$,

$$
H_{D}=\frac{\pi v_{\mathrm{pl}}}{L}\left[2 g P^{2}+2 \sum_{n=1}^{\infty} \alpha(-n) \alpha(n)\right]
$$

from which one gets that the partition function, at the strongly coupled fixed point

$$
\mathbf{Z}_{D}[\varphi]=\operatorname{Tr}\left[e^{-\beta H_{D}}\right]=\frac{1}{\eta(q)} \sum_{k \in Z} \exp \left[-\beta \frac{g \pi v_{\mathrm{pl}}}{L}\left(-\frac{\varphi}{2 \pi}+k\right)^{2}\right]
$$

with $\eta(x)=\prod_{n=1}^{\infty}\left(1-x^{n}\right)$, and $q=\exp \left[-\beta \frac{\pi v_{\mathrm{pl}}}{L}\right]$,

leading to the well-known sawtooth-like dependence on $\varphi$ of the Josephson current as $\beta \rightarrow \infty$ [7], since

$$
I[\varphi]=-\lim _{\beta \rightarrow \infty} \frac{1}{\beta} \frac{\partial \ln \mathbf{Z}_{D}[\varphi]}{\partial \varphi} \propto \varphi-[\varphi]
$$

of course, $[\varphi]$ is the integer part of $\varphi$ (in units of $2 \pi$ ).

At strong coupling, the degeneracy among the minima of the boundary term is removed by the magnetic energy, $E_{k}^{(0)}=\frac{g \pi v_{\mathrm{pl}}}{L}\left(-\frac{\varphi}{2 \pi}+k\right)^{2}$, selecting only one eigenvalue of the zero mode of the field $\Phi(x, t)$, except when $\varphi=\pi+2 \pi k$, where $E_{k}^{(0)}=E_{k+1}^{(0)}$. When these two levels are confined away from the others, the rf-SQUID may operate as a qubit [11]. For $L>L_{*}$, however, the oscillatory modes of the plasmon field determine a renormalization of the parameters of the effective two-level system, as it will be discussed in the following.

\subsection{Renormalization of the Josephson current for the rf-SQUIDs}

At strong coupling and for $\varphi=\pi$, the ground state is twofold degenerate, that is, $E_{0}^{(0)}=E_{1}^{(0)}$. This degeneracy is removed by phase slips (instantons), of amplitude $\sqrt{2} \pi$, connecting the ground states.

In order to describe phase slips within the framework of the two-boundary sine Gordon model, one has to introduce the dual field of $\Phi(x, t), \Theta(x, t)$, defined 
by

$$
\frac{\partial \Phi(x, t)}{\partial x}=\frac{v_{\mathrm{pl}}}{2 g} \frac{\partial \Theta(x, t)}{\partial t} ; \frac{2 g}{v_{\mathrm{pl}}} \frac{\partial \Phi(x, t)}{\partial t}=\frac{\partial \Theta(x, t)}{\partial x}
$$

Due to the duality between $\Phi$ and $\Theta$, the effective Hamiltonian given in Eq.(33) may be usefully rewritten as

$$
H_{D}=\frac{1}{4 \pi(2 g)} \int_{0}^{L} d x\left[\frac{1}{v_{\mathrm{pl}}}\left(\frac{\partial \Theta}{\partial t}\right)^{2}+v_{\mathrm{pl}}\left(\frac{\partial \Theta}{\partial x}\right)^{2}\right] .
$$

From the algebra of the bosonic fields, one easily derives [19] the commutation relations

$$
\left[\frac{\partial \Phi(x, t)}{\partial x}, \Theta(y, t)\right]=\left[\frac{\partial \Theta(x, t)}{\partial x}, \Phi(y, t)\right]=2 \pi i \delta(x-y)
$$

In order to construct the leading correction to the Dirichlet fixed point, one may use the Delayed Evaluation of Boundary Conditions (DEBC) approach, introduced in Ref.[19]: for this purpose, one has first to consider the most general primary field of the bulk theory, namely, a vertex operator of the form $V_{n, \tilde{n}}(x, t)=: \exp \left[i \frac{n}{\sqrt{2}} \Phi(x, t)+i \frac{\tilde{n}}{\sqrt{2}} \Theta(x, t)\right]:$, and then to obtain the boundary perturbation, $V_{n, \tilde{n}}^{(B)}(t)$, by evaluating it at $x=L$, using the pertinent boundary conditions for the fields $\Phi(x, t)$ and $\Theta(x, t)$. Since $\Phi(x, t)$ obeys to the Dirichlet boundary conditions at $x=0$ and at $x=L$, it will not contribute to $V_{n, \tilde{n}}^{(B)}(t)$. At variance, $\Theta(x, t)$, obeys to the Neumann boundary conditions at both boundaries; namely, one has that

$$
\frac{\partial \Theta(0, t)}{\partial x}=\frac{\partial \Theta(L, t)}{\partial x}=0
$$

implying the following mode expansion for $\Theta(x, t)$

$$
\Theta(x, t)=\theta_{0}+\frac{2 \pi u t}{L} 2 g P+2 i \sqrt{g} \sum_{n \neq 0} \frac{\alpha(n)}{n} \cos \left[\frac{\pi n}{L} x\right] e^{-i k_{n} t}
$$

with $\theta_{0}=\left(q_{R}-q_{L}\right) / \sqrt{2}$ and $k_{n}=\pi n / L$. 
As a consequence of Eq.(40), one finds

$$
\Theta(i \tau)=\Theta(L, i \tau)=\theta_{0}+\frac{2 \pi i u \tau}{L} 2 g P+i 2 \sqrt{g} \sum_{n \neq 0} \frac{\alpha(n)}{n}(-1)^{n} e^{-\frac{\pi n}{L} \tau}
$$

and thus, the generic boundary perturbation at the strongly coupled fixed point, $\tilde{H}_{\tilde{B}}$, may be written as a linear combination of the vertices $V_{\tilde{n}}=V_{0, \tilde{n}}^{(B)}$ as

$$
\tilde{H}_{\tilde{B}}=-\sum_{\tilde{n}} \lambda_{\tilde{n}}: e^{i \frac{\tilde{n}}{\sqrt{2}} \Theta(L)}:
$$

At the Dirichlet fixed point, the partition function $\tilde{Z}_{D}[\varphi]$, including the boundary interaction in Eq.(42), is written as

$$
\tilde{\mathbf{Z}}_{D}[\varphi]=\operatorname{Tr}\left[e^{-\beta H_{D}} \mathbf{T}_{\tau} \exp \left\{\sum_{\tilde{n} \neq 0} \int_{0}^{\beta} d \tau \lambda_{\tilde{n}}: e^{i \frac{\tilde{n}}{\sqrt{2}} \Theta(i \tau)}:\right\}\right]
$$

with $\Theta(i \tau)=\Theta(L, i \tau)$.

Eq.(43) reduces to Eq.(34) when all the $\lambda_{n}$ 's are set to zero.

Following the same steps used in section (2.1), one may derive the scaling dimension of $V_{\tilde{n}}, h_{\tilde{n}}$, from the large- $L$-limit of the two point correlation function; namely,

$$
\left\langle\mathbf{T}_{\tau}\left[V_{\tilde{n}}(i \tau) V_{-\tilde{n}}\left(i \tau^{\prime}\right)\right]\right\rangle_{(0)} \approx\left|\frac{\pi v_{\mathrm{pl}}}{L}\right| \tau-\tau^{\prime}||^{-2 g \tilde{n}^{2}}
$$

where $\langle\ldots\rangle_{(0)}$ denotes thermal averaging defined by the free Hamiltonian with Dirichlet boundary conditions for $\Phi(x, t)$ at both boundaries.

Eq.(44) implies that $h_{\tilde{n}}=g \tilde{n}^{2}$ and that the leading perturbation at the strongly coupled fixed point is given by

$$
H_{\tilde{B}}=-Y\left[\left[: e^{i \frac{\Theta(L)}{\sqrt{2}}}:+: e^{-i \frac{\Theta(L)}{\sqrt{2}}}:\right]\right.
$$

The physical meaning of the operators $V_{\tilde{n}}$ may be inferred from the commu- 
tation relation

$$
\left[P,: e^{i \tilde{n} \Theta(i \tau)}:\right]=-\frac{1}{2 \pi} \int_{0}^{L} d x\left[\frac{\partial \Phi(x, i \tau)}{\partial x},: e^{i \frac{\tilde{n}}{\sqrt{2}} \Theta(t)}:\right]=\frac{\tilde{n}}{\sqrt{2}}: e^{i \frac{\tilde{n}}{\sqrt{2}} \Theta(i \tau)}:
$$

which shows that $V_{\tilde{n}}$ changes the eigenvalue of $P$ by $\tilde{n} / \sqrt{2}$. Thus, the boundary fields : $e^{ \pm \frac{i}{\sqrt{2}} \Theta(i \tau)}$ : describe instanton/antiinstanton trajectories between the two ground states that are degenerate at $\varphi=\pi$ and the parameter $Y$ may be interpreted as the instanton fugacity.

A dimensionless running coupling $Y_{\text {Inst }}(L)$ is defined as

$$
Y_{\text {Inst }}(L)=Y L^{1-g}
$$

From Eq.(47), one sees that, for $g<1$, (i.e., when the Neumann fixed point is stable), instantons are a relevant perturbation, since $Y_{\text {Inst }}(L)$ scales as $\left(\frac{L}{L_{*}}\right)^{1-g}$. However, the scaling ceases to be valid as $Y_{\text {Inst }}(L) \sim 1$.

For $g>1$, using the boundary perturbation operator introduced in Eq. (45), it is straightforward to compute the leading corrections to the Josephson current. For instance, when $\varphi \sim 0$, one may approximate the partition function in Eq. (43) as

$$
\begin{aligned}
& \tilde{\mathbf{Z}}_{D}[\varphi] \approx \frac{\exp \left[-\beta \frac{g v_{\mathrm{pl}}}{4 \pi L} \varphi^{2}\right]}{\prod_{n>0}\left[1-q^{n}\right]}\left\langle P_{0},\{0\}\right|\{1+ \\
& \left.2 Y^{2} \int_{0}^{\beta} d \tau_{1} d \tau_{2} \mathbf{T}_{\tau}\left[: \cos \left(\frac{1}{\sqrt{2}} \Theta\left(i \tau_{1}\right)\right):: \cos \left(\frac{1}{\sqrt{2}} \Theta\left(i \tau_{2}\right)\right):\right]\right\}\left|P_{0},\{0\}\right\rangle
\end{aligned}
$$

By inserting in Eq.(48) the explicit form of the correlator

$$
\left\langle P_{0},\{0\}\left|\mathbf{T}_{\tau}\left[: \cos \left(\frac{\Theta\left(i \tau_{1}\right)}{\sqrt{2}}\right):: \cos \left(\frac{\Theta\left(i \tau_{2}\right)}{\sqrt{2}}\right):\right]\right| P_{0},\{0\}\right\rangle=\frac{\cosh \left[\frac{g v_{\mathrm{pl}} \varphi}{2 L}\left(\tau_{1}-\tau_{2}\right)\right]}{\left[2 \sinh \left[\frac{\pi v_{\mathrm{pl}}}{2 L}\left|\tau_{1}-\tau_{2}\right|\right]\right]^{2 g}},(49)
$$

one gets the partition function $\mathbf{Z}[\varphi]$

$$
\tilde{\mathbf{Z}}_{D}[\varphi] \approx \frac{\exp \left[-\beta \frac{g v_{\mathrm{pl}}}{4 \pi L} \varphi^{2}\right]}{\prod_{n>0}\left[1-q^{n}\right]}\left\{1+\frac{\beta L}{\pi v_{\mathrm{pl}}} Y^{2} \Gamma[1-2 g]\left\{\frac{\Gamma\left[g\left(1-\frac{\varphi}{2 \pi}\right)\right]}{\Gamma\left[1-g\left(1+\frac{\varphi}{2 \pi}\right)\right]}+\frac{\Gamma\left[g\left(1+\frac{\varphi}{2 \pi}\right)\right]}{\Gamma\left[1-g\left(1-\frac{\varphi}{2 \pi}\right)\right]}\right\}\right\}
$$


Computing the logarithmic derivative of Eq.(50) with respect to $\varphi$ and dividing it by $-\beta$, one obtains

$$
\begin{aligned}
& I[\varphi] \approx \frac{e^{*} g v_{\mathrm{pl}}}{2 \pi L} \varphi-e^{*} \frac{2 L \Gamma[1-2 g]}{2 \pi v_{\mathrm{pl}}} Y^{2} \frac{\partial}{\partial \varphi}\left\{\frac{\Gamma\left[g\left(1-\frac{\varphi}{2 \pi}\right)\right]}{\Gamma\left[1-g\left(1+\frac{\varphi}{2 \pi}\right)\right]}+\frac{\Gamma\left[g\left(1+\frac{\varphi}{2 \pi}\right)\right]}{\Gamma\left[1-g\left(1-\frac{\varphi}{2 \pi}\right)\right]}\right\} \\
& \approx\left[\frac{e^{*} g v_{\mathrm{pl}}}{2 \pi L}-Y^{2} G[g]\right] \varphi
\end{aligned}
$$

with

$$
G[g]=e^{*} \frac{2 L \Gamma[1-2 g]}{4 \pi^{2} v_{\mathrm{pl}}} \frac{\partial^{2}}{\partial \varphi^{2}}\left\{\frac{\Gamma\left[g\left(1-\frac{\varphi}{2 \pi}\right)\right]}{\Gamma\left[1-g\left(1+\frac{\varphi}{2 \pi}\right)\right]}+\frac{\Gamma\left[g\left(1+\frac{\varphi}{2 \pi}\right)\right]}{\Gamma\left[1-g\left(1-\frac{\varphi}{2 \pi}\right)\right]}\right\}_{\varphi=0}
$$

For small $\varphi, Y, H_{\tilde{B}}$ provides a small correction to the slope of the Josephson current. As we shall see in detail in the following subsection, for $\varphi=\pi$ and $g>1$, instantons at the Dirichlet fixed point remove the degeneracy between the minima and smooth the edges in the sawtooth-like Josephson current. For $g<1$, of course, instantons are irrelevant operators.

\subsection{Instanton constribution to the Josephson current at the Dirichlet fixed point for $\varphi=\pi$.}

In the previous section, it has been shown that, for $\varphi=\pi$, the state $\left.P_{0},\{0\}\right\rangle$ becomes degenerate with $\left.P_{1},\{0\}\right\rangle$. This corresponds to a discontinuous jump of $e^{*} g v_{\mathrm{pl}} / L$ in the Josephson current, between the values corresponding to the states that are degenerate at $\varphi=\pi$. Since instantons connecting the two states should remove the degeneracy, for $Y \neq 0$ one should expect that the Josephson current becomes a continuous function of $\varphi$ at $\varphi=\pi$.

In order to compute the $Y$-dependent corrections to the ground state energy, it is useful to partition the Hilbert space into subspaces on which $H_{\tilde{B}}$ is diagonal. If $\left|P_{k}, \Psi\right\rangle$ denotes a generic state in the sector corresponding to the eigenvalue $P_{k}$ of the zero-mode operator, one defines the states belonging to the above subspaces as

$$
| \pm, \Psi\rangle=\frac{1}{\sqrt{2}}\left[\left|P_{0}, \Psi\right\rangle \pm\left|P_{1}, \Psi\right\rangle\right]
$$


Thus, the partition function at $\varphi=\pi$ may be approximated as

$$
\tilde{\mathbf{Z}}_{D}[\pi]=\operatorname{Tr}_{|-, \Psi\rangle}\left\{e^{-\beta H_{D}} \mathbf{T}_{\tau} \exp \left[\int_{0}^{\beta} d \tau \tilde{H}_{B}(i \tau)\right]\right\}+\operatorname{Tr}_{|+, \Psi\rangle}\left\{e^{-\beta H_{D}} \mathbf{T}_{\tau} \exp \left[\int_{0}^{\beta} d \tau \tilde{H}_{B}(i \tau)\right]\right\},
$$

where $\operatorname{Tr}_{|\mp, \Psi\rangle}$ denotes tracing over the sector of the Fock space spanned by the states $| \pm, \Psi\rangle$.

At low temperature, Eq.(53) may be approximated as

$$
\begin{aligned}
& \tilde{\mathbf{Z}}_{D}[\pi] \approx\left\langle-,\{0\}\left|e^{-\beta H_{D}} \mathbf{T}_{\tau} \exp \left[\int_{0}^{\beta} d \tau \tilde{H}_{B}(i \tau)\right]\right|-,\{0\}\right\rangle \\
& +\left\langle+,\{0\}\left|e^{-\beta H_{D}} \mathbf{T}_{\tau} \exp \left[\int_{0}^{\beta} d \tau \tilde{H}_{B}(i \tau)\right]\right|+,\{0\}\right\rangle,
\end{aligned}
$$

where $| \pm, 0\rangle$ are, of course, the ground states of both subspaces. By expanding $\mathbf{T}_{\tau} \exp \left[\int_{0}^{\beta} d \tau \tilde{H}_{B}(i \tau)\right]$ in a power series of $Y$, one gets

$$
\begin{aligned}
& \left\langle \pm,\{0\}\left|e^{-\beta H_{D}} \mathbf{T}_{\tau} \exp \left[\int_{0}^{\beta} d \tau \tilde{H}_{B}(i \tau)\right]\right| \pm,\{0\}\right\rangle= \\
& \exp \left[-\beta \frac{g \pi v_{\mathrm{pl}}}{4 L}\right] \sum_{m=0}^{\infty} \frac{1}{m !} \int_{0}^{\beta} d \tau_{1} \ldots \int_{0}^{\beta} d \tau_{m}\left\langle \pm,\{0\}\left|\mathbf{T}_{\tau}\left[\tilde{H}_{B}\left(i \tau_{1}\right) \ldots \tilde{H}_{B}\left(i \tau_{m}\right)\right]\right| \pm,\{0\}\right\rangle
\end{aligned}
$$

When computing the partition function, one has to sum over contributions from connected, as well as from disconnected diagrams. By applying Wick's theorem, one obtains

$$
\begin{aligned}
& \left\langle \pm,\{0\}\left|\mathbf{T}_{\tau}\left[\tilde{H}_{B}\left(i \tau_{1}\right) \ldots \tilde{H}_{B}\left(i \tau_{m}\right)\right]\right| \pm,\{0\}\right\rangle=\prod_{j=1}^{M}\left\langle \pm,\{0\}\left|\tilde{H}_{B}\left(i \tau_{1}\right)\right| \pm,\{0\}\right\rangle+ \\
& \sum_{j_{1} \neq j_{2}=1}^{M}\left\langle \pm,\{0\}\left|\mathbf{T}_{\tau}\left[\tilde{H}_{B}\left(i \tau_{j_{1}}\right) \tilde{H}_{B}\left(i \tau_{j_{2}}\right)\right]\right| \pm,\{0\}\right\rangle \prod_{i \neq j_{1}, j_{2}}\left\langle \pm,\{0\}\left|\tilde{H}_{B}\left(i \tau_{i}\right)\right| \pm,\{0\}\right\rangle+\ldots
\end{aligned}
$$

Since $\left\langle \pm, \mathrm{GS}\left|\tilde{H}_{B}(i \tau)\right| \pm, \mathrm{GS}\right\rangle= \pm Y$, it is straightforward to evaluate, using Eqs.(55, 56$)$, the leading $Y$-dependent contribution to the energy of the states 
$\pm,\{0\}\rangle$; namely $E_{ \pm}$is given by

$$
E_{ \pm}=\frac{g \pi v_{\mathrm{pl}}}{4 L} \mp Y-\frac{L}{\pi v_{\mathrm{pl}}} Y^{2} \Gamma[1-2 g]\left\{\frac{\Gamma\left[\frac{g}{2}\right]}{\Gamma\left[1-\frac{3}{2} g\right]}+\frac{\Gamma\left[\frac{3}{2} g\right]}{\Gamma\left[1-\frac{g}{2}\right]}\right\},
$$

and, thus, even to the first order in $Y$, instantons remove the degeneracy between the ground states.

The observable consequence of the removal of the ground state degeneracy is the smoothing down of the Josephson current, which becomes a continuous function of the applied flux $\varphi$ when it crosses the value $\varphi=\pi$.

In order to see this effect, one has to resum over instanton contributions to the partition function, by setting $\varphi=\pi+\epsilon$, with $\epsilon / \pi \ll 1$. In order to obtain a meaningful result, one has first to sum over the instanton contributions, and then to compute the leading corrections in $\epsilon$.

When $\varphi=\pi+\epsilon$, the low temperature partition function may be approximated as

$$
\tilde{\mathbf{Z}}_{D}[\pi+\epsilon]=\sum_{a= \pm} \operatorname{Tr}_{|a, \Psi\rangle}\left\{e^{-\beta H_{D}} \mathbf{T}_{\tau} \exp [-\delta S[Y, \epsilon]]\right\},
$$

with

$$
\delta S[Y, \epsilon]=2 Y \int_{0}^{\beta} d \tau \cos \left[\frac{\Theta(i \tau)}{\sqrt{2}}\right]+\beta\left[\frac{g v_{\mathrm{pl}}}{L} \epsilon P+\frac{g \epsilon^{2}}{4 \pi L}\right] .
$$

When $\epsilon=0$, one obtains the result of the previous section. When computing the leading corrections in $\epsilon$, one has to notice that $P$ mixes the two subspaces labeled by \pm ; namely

$$
\langle \pm, \Psi|P| \mp, \Psi\rangle=\mp \frac{1}{2 \sqrt{2}}
$$

Thus, the leading $\epsilon$-dependent contribution to Eq.(159), $\delta \tilde{\mathbf{Z}}_{D}[\epsilon]$, appears only to the order $\epsilon^{2}$, and is given by

$$
\delta \tilde{\mathbf{Z}}_{D}[\epsilon]=\tilde{\mathbf{Z}}_{D}[\pi] e^{-\beta \frac{g v_{\mathrm{pl}}}{4 \pi L} \epsilon^{2}} \sum_{a= \pm} \sum_{m=0}^{\infty}\left\{\frac{(m+1)(m+2)}{(m+2) !}\left(\frac{g v_{\mathrm{pl}} \epsilon}{2 L}\right)^{2} \times\right.
$$




$$
\begin{aligned}
& \left.\left\langle a,\{0\}\left|(\beta P)^{2}\right| a,\{0\}\right\rangle \prod_{i=1}^{m} \int_{0}^{\beta} d \tau_{i}\left\langle a,\{0\}\left|\tilde{H}_{B}\left(i \tau_{j}\right)\right| a,\{0\}\right\rangle\right\} \\
& =\tilde{\mathbf{Z}}_{D}[\pi] e^{-\beta \frac{g v_{\mathrm{pl}}}{4 \pi L} \epsilon^{2}} \sum_{m=0}^{\infty}\left\{\frac{(m+1)(m+2)}{(m+2) !}(\beta Y)^{m+1}\left[1+(-1)^{m}\right]\left(\frac{g v_{\mathrm{pl}}}{2 L}\right)^{2} \frac{\beta \epsilon^{2}}{2 Y}\right\} \\
& =\tilde{\mathbf{Z}}_{D}[\pi] \sum_{a= \pm} \exp \left[a \beta\left(Y+\left(\frac{g v_{\mathrm{pl}}}{2 L}\right)^{2} \frac{\epsilon^{2}}{2 Y}\right)\right]
\end{aligned}
$$

To the leading order in $\epsilon$, the ground state energy derived from Eq.(61) is given by

$$
E_{\mathrm{GS}}[\epsilon]=E_{\mathrm{GS}}[\epsilon=0]-Y-\left(\frac{g v_{\mathrm{pl}}}{2 L}\right)^{2} \frac{\beta \epsilon^{2}}{2 Y}+\frac{g v_{\mathrm{pl}}}{4 \pi L} \epsilon^{2}
$$

Eq. (62) may be regarded as the lowest order term in the expansion of the function $-\sqrt{Y^{2}+\left(\frac{g v_{\mathrm{pl}} \epsilon}{2 L}\right)^{2}}$. Although our approach allows to compute, in principle, also higher-order corrections, for our purposes it is enough to notice that, already to the leading order in $\epsilon$, one gets that the current behaves as a continuous straight line, with no sharp, discontinous, jumps, at $\epsilon=0$ (i.e., $\varphi=\pi)$.

$$
I=e^{*} \frac{\partial E_{\mathrm{GS}}[\epsilon]}{\partial \epsilon} \approx \frac{g v_{\mathrm{pl}}}{2 \pi L} \epsilon-\left(\frac{g v_{\mathrm{pl}}}{2 L}\right)^{2} \frac{\epsilon}{Y}
$$

Eq.(63) is not only consistent with the results of previous analyses of rfSQUIDS [7,34], but also with a strong coupling expansion of the ground state energy of the two-boundary sine Gordon model derived in Ref.[15] using the Bethe ansatz approach, once one of the two couplings is set to $\infty$, and a strong coupling expansion in inverse powers of the other coupling has been performed. This makes us confident that the smoothing effect of the Josephson current due to instantons -exactly computed in Ref.[15] for $g=1$ - is effective for any value of the Luttinger parameter $g$.

\section{The dc-SQUID}

A dc-SQUID is realized as a superconducting loop, interrupted by two Josephson junctions, of nominal values $E_{J, 1}$ and $E_{J, 2}$. It is possible to generalize to 


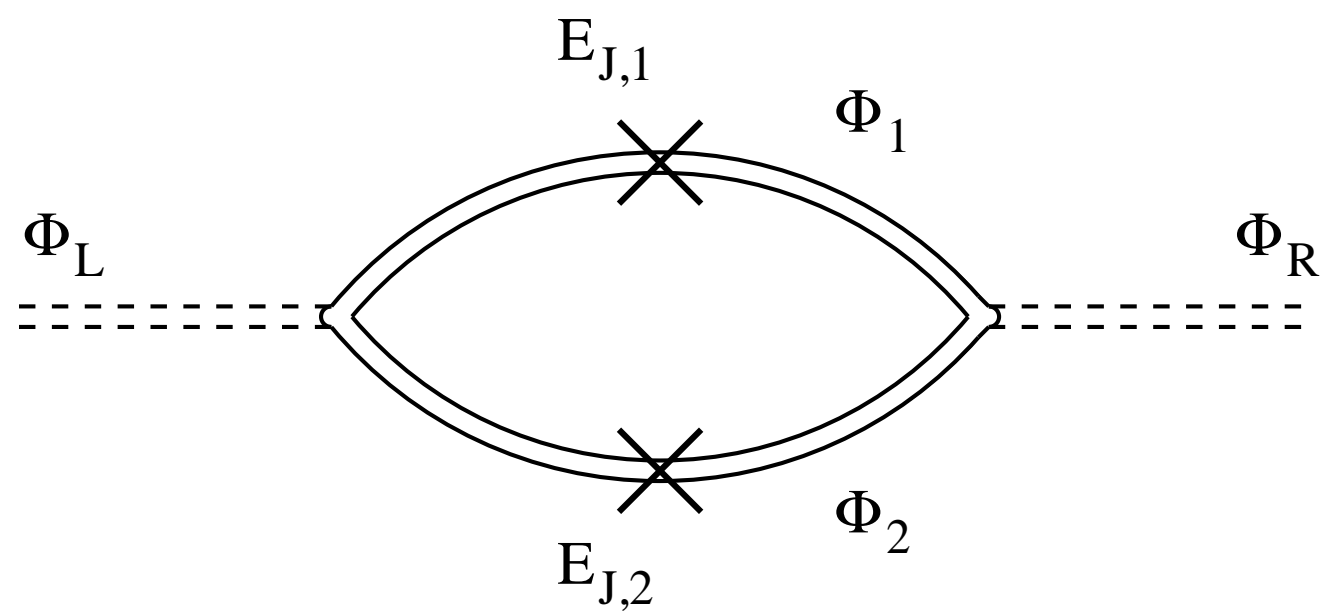

Fig. 1. Sketch of the dc-SQUID device with the leads.

the dc-SQUID the renormalization group analysis developed so far for the rfSQUID, obtaining that, also in this case, the only alleged fixed points are a weakly coupled (Neumann), and a strongly coupled (Dirichlet) one.

The device is drawn in Fig.(11): it is made out of two superconducting wires, connected by two Josephson junctions, of nominal values $E_{J, 1}, E_{J, 2}$, with a magnetic flux $\Phi$ piercing the loop. $\Phi_{1}(x), \Phi_{2}(x)$ are the plasmon fields in the arms connecting the two junctions.

When, in the following section, we investigate the transport properties of a dc-SQUID, the leads to which the device drawn in Fig.(11) is connected, are described by two additional plasmon fields $\Phi_{L}(x)$ and $\Phi_{R}(x)$.

The Hamiltonian describing the dynamics of $\Phi_{1}(x), \Phi_{2}(x)$ is given by

$$
H_{d c, 0}=\frac{g}{2 \pi} \sum_{j=1,2} \int_{-L}^{L} d x\left[\frac{1}{v_{\mathrm{pl}}}\left(\frac{\partial \Phi_{j}}{\partial t}\right)^{2}+v_{\mathrm{pl}}\left(\frac{\partial \Phi_{j}}{\partial x}\right)^{2}\right]
$$

while the Josephson energy of the two junctions is described now by the boundary interaction Hamiltonian $H_{d c, J}$ given by

$$
H_{d c, J}=-E_{J, 1} \cos \left[\Phi_{2}(L)-\Phi_{1}(L)+\frac{\varphi}{2}\right]-E_{J, 2} \cos \left[\Phi_{2}(-L)-\Phi_{1}(-L)-\frac{\varphi}{2}\right],
$$

with $\varphi=\Phi / \Phi_{0}^{*}$.

Again, it is most convenient to introduce the even- and odd-fields, $\Phi_{j, e / o}(x)$, 
as

$$
\Phi_{j, e / o}(x)=\frac{1}{\sqrt{2}}\left[\Phi_{j}(x) \pm \Phi_{j}(-x)\right] \quad ; 0 \leq x \leq L
$$

By definition, the $\Phi_{j, e}(x)$ 's obey to Neumann boundary conditions at $x=0$, while the $\Phi_{j, o}(x)$ 's obey to Dirichlet boundary conditions; namely, one has

$$
\frac{\partial \Phi_{1, e}(0, t)}{\partial x}=\frac{\partial \Phi_{2, e}(0, t)}{\partial x}=0 ; \Phi_{1, o}(0, t)=\Phi_{2, o}(0, t)=0
$$

The boundary Hamiltonian $H_{d c, J}$ gets contributions only from two linear combinations of the fields $\Phi_{e}, \Phi_{o}$. Defining

$$
X(x)=\frac{1}{\sqrt{2}}\left[\Phi_{2, e}(x)-\Phi_{1, e}(x)\right] ; \xi(x)=\frac{1}{\sqrt{2}}\left[\Phi_{2, o}(x)-\Phi_{1, o}(x)\right]
$$

it is easy to realize that the device is described by the reduced Hamiltonian $H_{d c}$ given by

$$
\begin{aligned}
& H_{d c}=\frac{g}{2 \pi} \int_{0}^{L} d x\left\{\left[\frac{1}{v_{\mathrm{pl}}}\left(\frac{\partial X}{\partial t}\right)^{2}+v_{\mathrm{pl}}\left(\frac{\partial X}{\partial x}\right)^{2}\right]+\left[\frac{1}{v_{\mathrm{pl}}}\left(\frac{\partial \xi}{\partial t}\right)^{2}+v_{\mathrm{pl}}\left(\frac{\partial \xi}{\partial x}\right)^{2}\right]\right\} \\
& -E_{J, 1} \cos \left[X(L)+\xi(L)+\frac{\varphi}{2}\right]-E_{J, 2} \cos \left[X(L)-\xi(L)-\frac{\varphi}{2}\right]
\end{aligned}
$$

$H_{d c}$ in Eq.(69) may be regarded as the two-field generalization of the two boundary sine Gordon Hamiltonian describing the properties of an rf-SQUID. By construction, at the inner boundary, $x=0, X(x)$ obeys to Neumann boundary conditions, $\frac{\partial X(0, t)}{\partial x}=0$, while $\xi(x)$ obeys to Dirichlet boundary conditions, $\xi(0, t)=0$. The boundary conditions at the outer boundary, $x=L$, are obtained by requiring that the energy functional is conserved; namely, by

$$
\begin{aligned}
& \frac{g v_{\mathrm{pl}}}{\pi} \frac{\partial X(L, t)}{\partial x}+E_{J, 1} \sin \left[X(L, t)+\xi(L, t)+\frac{\varphi}{2}\right]+E_{J, 2} \sin \left[X(L, t)-\xi(L, t)-\frac{\varphi}{2}\right]=0, \\
& \frac{g v_{\mathrm{pl}}}{\pi} \frac{\partial \xi(L, t)}{\partial x}+E_{J, 1} \sin \left[X(L, t)+\xi(L, t)+\frac{\varphi}{2}\right]-E_{J, 2} \sin \left[X(L, t)-\xi(L, t)-\frac{\varphi}{2}\right]=0 \quad .(70)
\end{aligned}
$$

For $E_{J, 1}=E_{J, 2}=0, X$ and $\xi$ obey to Neumann boundary conditions at $x=L$ : $\frac{\partial X(L, t)}{\partial x}=\frac{\partial \xi(L, t)}{\partial x}=0$. For $E_{J, 1}, E_{J, 2} \rightarrow \infty$, the fields obey to Dirichlet boundary 
conditions, $X(L, t)+\xi(L, t)-\frac{\varphi}{2}=0(\bmod 2 \pi), X(L, t)-\xi(L, t)+\frac{\varphi}{2}=0(\bmod$ $2 \pi)$.

In the following, we shall use $H_{d c}$ to derive the renormalization of the Josephson energies and the functional form of the Josephson current in both the weakly coupled and the strongly coupled regimes accessible to a dc-SQUID.

\subsection{The weakly coupled fixed point}

At the fixed point $E_{J, 1}=E_{J, 2}=0, X(x)$ obeys to Neumann boundary conditions at both boundaries, while $\xi(x)$ satisfies mixed boundary conditions (namely, Dirichlet boundary conditions at $x=0$ and Neumann boundary conditions at $x=L)$. The mode expansion of the field $X(x, t)$ is, then, given by

$$
X(x, t)=q_{X}+\frac{\pi v_{\mathrm{pl}} t}{L} \frac{\tilde{P}_{X}}{g}+i \sqrt{\frac{1}{g}} \sum_{n \neq 0} \frac{\alpha_{X}(n)}{n} \cos \left(k_{n} x\right) \exp \left(-i k_{n} v_{\mathrm{pl}} t\right)
$$

with $\left[\alpha_{X}(n), \alpha_{X}\left(n^{\prime}\right)\right]=n \delta_{n+n^{\prime}, 0} ; q_{X}$ is the constant zero-mode operator and $k_{n}=\frac{\pi}{L} n . \tilde{P}_{X}$ is, of course, the "dual" zero-mode operator, whose eigenvalues are the winding numbers.

The mixed boundary conditions for the field $\xi(x, t)$ are satisfied by setting

$$
\xi(x, t)=-\sqrt{\frac{1}{g}} \sum_{n \neq 0} \frac{a_{\xi}(n)}{n} \sin \left[K_{n} x\right] e^{i K_{n} v_{\mathrm{p} 1} t},
$$

with $\left[\alpha_{\xi}(n), \alpha_{\xi}\left(n^{\prime}\right)\right]=n \delta_{n+n^{\prime}, 0}$, and $K_{n}=\frac{\pi}{L}\left(n+\frac{1}{2}\right)$.

By taking into account Eqs.(71,72), the boundary interaction may be normal ordered as in section 2. In particular, one obtains

$$
H_{d c, J}=-\bar{E}_{J, 1}: \cos \left[X(L)+\xi(L)+\frac{\varphi}{2}\right]:-\bar{E}_{J, 2}: \cos \left[X(L)-\xi(L)-\frac{\varphi}{2}\right]:
$$

with $\bar{E}_{J, j}=\left(\frac{2 \pi a}{L}\right)^{\frac{1}{g}} E_{J, j}, j=1,2$.

Again, the scaling equations for the pertinent running couplings are obtained from the requirement that the partition function $\mathbf{Z}$ is independent of the cutoff. $\mathbf{Z}$ is given by

$$
\mathbf{Z}=\int \mathbf{D} X \mathbf{D} \xi\left\{e^{-S_{0}} \mathbf{T}_{\tau}\left[e^{-S_{B}}\right]\right\}
$$


with

$$
\begin{aligned}
& S_{0}=\frac{g}{2 \pi} \int_{0}^{\beta} d \tau \int_{0}^{L} d x\left[\frac{1}{v_{\mathrm{pl}}}\left(\frac{\partial X(x, i \tau)}{\partial \tau}\right)^{2}+v_{\mathrm{pl}}\left(\frac{\partial X(x, i \tau)}{\partial x}\right)^{2}\right] \\
& +\frac{g}{2 \pi} \int_{0}^{\beta} d \tau \int_{0}^{L} d x\left[\frac{1}{v_{\mathrm{pl}}}\left(\frac{\partial \xi(x, i \tau)}{\partial \tau}\right)^{2}+v_{\mathrm{pl}}\left(\frac{\partial \xi(x, i \tau)}{\partial x}\right)^{2}\right]
\end{aligned}
$$

and

$$
\begin{aligned}
& S_{B}=-\frac{\bar{E}_{J, 1}}{2} \int_{0}^{\beta} d \tau\left[e^{i \varphi} V_{1,1}(i \tau)+e^{-i \varphi} V_{-1,-1}(i \tau)\right] \\
& -\frac{\bar{E}_{J, 2}}{2} \int_{0}^{\beta} d \tau e^{i \varphi}\left[V_{1,-1}(i \tau)+e^{-i \varphi} V_{-1,1}(i \tau)\right]
\end{aligned}
$$

while the vertex operators $V_{a, b}(\tau)$ are now given by

$$
V_{a, b}(i \tau)=: \exp \left\{i[b X(i \tau)+a \xi(i \tau)]-a \frac{\varphi}{2}\right\}: \quad ; \quad a, b= \pm 1
$$

To determine the scaling dimensions of the relevant operators, one needs to compute the correlators of the vertices written in Eq.(77). They can be computed as discussed in appendix B; however, for the purpose of deriving the perturbative RG equations, one only needs the short-distance limit of the vertex correlators given by

$$
\left\langle\mathbf{T}_{\tau}\left[V_{a, b}(i \tau) V_{a^{\prime}, b^{\prime}}\left(i \tau^{\prime}\right)\right]\right\rangle \approx\left[\frac{\pi v_{\mathrm{pl}}}{L}\left|\tau-\tau^{\prime}\right|\right]^{\frac{\left(a a^{\prime}-1\right)}{g}} \delta_{b+b^{\prime}, 0}
$$

Eq.(178) shows that the scaling dimension of $V_{a, b}$ is $h_{a, b}=1 / g$.

Since $\xi(\tau)$ does not have a zero mode, the O.P.E.'s generate, just as it happens for an rf-SQUID, higher periodicity terms, according to

$$
V_{a, b}(i \tau) V_{a^{\prime}, b^{\prime}}\left(i \tau^{\prime}\right) \approx_{\tau^{\prime} \rightarrow \tau^{-}}\left[\frac{\pi v_{\mathrm{pl}}}{L}|\tau-\tau|\right]^{-h_{a, b}-h_{a^{\prime}, b^{\prime}}+h_{a+a^{\prime}, b+b^{\prime}}} V_{a+a^{\prime}, b+b^{\prime}}\left(i \tau^{\prime}\right)
$$


To the second order in the couplings $\bar{E}_{J, 1}, \bar{E}_{J, 2}$, Eq.(179) requires to add to $S_{B}$ an extra counterterm given by

$$
\delta S_{B}=-\lambda_{2} \int_{0}^{\beta} d \tau\left[V_{2,0}(i \tau)+V_{-2,0}(i \tau)\right] .
$$

The running coupling constants $G_{1}, G_{2}$ are now defined as

$$
G_{1}(L)=\left(\frac{L}{2 \pi a}\right)^{1-\frac{1}{g}} \frac{a E_{J, 1}}{v_{\mathrm{pl}}} \quad ; \quad G_{2}(L)=\left(\frac{L}{2 \pi a}\right)^{1-\frac{1}{g}} \frac{a E_{J, 2}}{v_{\mathrm{pl}}} .
$$

Since the vertices $V_{ \pm 2,0}(i \tau)=e^{ \pm i \varphi}: \exp [ \pm 2 i \xi(\tau)]$ : are less relevant than the $V_{ \pm 1, \pm 1}$ 's, they do not contribute to the evaluation of the leading terms of the perturbative $\beta$ functions for $G_{1}, G_{2}$,

$$
\frac{d \ln G_{1}(L)}{d \ln \left(L / L_{0}\right)}=\beta_{1}\left(G_{1}, G_{2}\right)=\left[1-\frac{1}{g}\right] G_{1}(L)
$$

and

$$
\frac{d \ln G_{2}(L)}{d \ln \left(L / L_{0}\right)}=\beta_{2}\left(G_{1}, G_{2}\right)=\left[1-\frac{1}{g}\right] G_{2}(L)
$$

As for the rf-SQUID, one finds that both couplings are irrelevant for $g<1$; namely, the Neumann fixed point is infrared stable, and the theory is perturbative in the couplings $\bar{E}_{J, 1}, \bar{E}_{J, 2}$.

When $g>1$, instead, both couplings $G_{j}(L)$ increase, when $L$ increases, as $\left(L / L_{0}\right)^{1-\frac{1}{g}}$. Thus, just as for the rf-SQUID, the theory becomes nonperturbative as soon as $L \sim L_{*}$, with $L_{*} \sim 2 \pi a\left(\frac{v_{\mathrm{pl}}}{a E_{J, \min }}\right)^{\frac{g}{g-1}}$, being $E_{J \text {,min }}$ the smaller coupling, between $E_{J, 1}$ and $E_{J, 2}$.

To evaluate the Josephson current for $g<1$, we compute the partition function for finite $\varphi$. The result is

$$
\begin{aligned}
& \mathbf{Z}[\varphi]=\mathbf{Z}_{0}\left\langle\mathbf { T } _ { \tau } \left\{\operatorname { e x p } \left[\int _ { 0 } ^ { \beta } d \tau \left[\bar{E}_{J, 1} \cos \left[X(i \tau)+\xi(i \tau)+\frac{\varphi}{2}\right]\right.\right.\right.\right. \\
& \left.\left.\left.+\bar{E}_{J, 2} \cos \left[X(i \tau)-\xi(i \tau)-\frac{\varphi}{2}\right]\right]\right\}\right\rangle,
\end{aligned}
$$


with

$$
\mathbf{Z}_{0}=\prod_{n=0}^{\infty}\left\{\left[\frac{1}{1-q^{n+\frac{1}{2}}}\right]\left[\frac{1}{1-q^{n+1}}\right]\right\} \sum_{m \in Z} e^{-\beta \frac{2 g \pi v_{\mathrm{pl}}}{L} m^{2}}
$$

and $q=e^{-\beta \frac{\pi v_{\mathrm{pl}}}{L}}$.

Since the field $X(i \tau)$ has a constant zero-mode term, a perturbative expansion in the boundary interaction yields nonzero contributions to the partition function starting only from the second order in $\bar{E}_{J, 1}, \bar{E}_{J, 2}$, implying that, at low temperatures, the leading $\varphi$-dependence is given by

$$
\begin{aligned}
& \mathbf{Z}[\varphi]=\mathbf{Z}_{0}\left\langle\left\{1+\frac{1}{2} \int_{0}^{\beta} d \tau_{1} \int_{0}^{\beta} d \tau_{2} \mathbf{T}_{\tau}\left[H_{d c, J}\left(\tau_{1}\right) H_{d c, J}\left(\tau_{2}\right)\right]\right\}\right\rangle \\
& =\mathbf{Z}_{0}\left\langle\left\{1+\beta \bar{E}_{J, 1} \bar{E}_{J, 2} \cos (\varphi) \int_{0}^{\beta} d \tau \frac{1}{\left[2 \cosh \left(\frac{\pi u}{4 L} \tau\right)\right]^{\frac{2}{g}}}+\ldots\right\}\right. \\
& =\mathbf{Z}_{0}\left\langle\left\{1+\frac{2 L}{\pi u} \beta \bar{E}_{J, 1} \bar{E}_{J, 2} B \cos (\varphi)\right\}\right.
\end{aligned}
$$

with

$$
B=\frac{\sqrt{\pi} \Gamma\left[\frac{1}{g}\right]}{2^{\frac{2}{g}} \Gamma\left[1+\frac{1}{g}\right]}
$$

as a result, the Josephson current is given by

$$
I[\Phi]=-\frac{1}{c \Phi_{0}^{*}} \lim _{\beta \rightarrow \infty} \frac{\partial \ln \mathbf{Z}[\varphi]}{\partial \varphi}=e^{*} \frac{2 L}{\pi u} B \bar{E}_{J, 1} \bar{E}_{J, 2} \sin (\varphi)
$$

The functional form of $I[\Phi]$ is similar to Eq.(24), but it is now proportional to $\bar{E}_{J, 1} \bar{E}_{J, 2}$. For $g<1$, Eq. (87) is valid for any value of $L$, since the quantum corrections are perturbative in $\bar{E}_{J, 1} \bar{E}_{J, 2}$. When $g>1$, instead, higher harmonics operators become more and more relevant as $L$ increases, inducing a crossover to a sawtooth-like functional form of the Josephson current, as $L$ approaches $L_{*}$. 


\subsection{The strongly coupled fixed point}

In this subsection the strong coupling $\left(E_{J, j} /\left(2 \pi v_{\mathrm{pl}} / L\right) \ll 1\right)$ regime of a dcSQUID is analyzed and the explicit functional forms of the partition function and of the Josephson current are derived. To compute the partition function with Dirichlet-like boundary conditions at the outer boundary, $X(L, t)+$ $\left.\xi(L, t)+\frac{\varphi}{2}=0(\bmod 2 \pi)\right)$, and $\left.X(L, t)-\xi(L, t)-\frac{\varphi}{2}=0(\bmod 2 \pi)\right)$, one notices that

$$
X(L, t)=\pi\left(n_{1}+n_{2}\right) \quad ; \quad \xi(L, t)-\frac{\varphi}{2}=\pi\left(n_{1}-n_{2}\right)
$$

with $n_{1}, n_{2}$ relative integers.

Eqs.(88), together with the boundary conditions at the inner boundary, lead to

$$
\xi(x, t)=-\frac{2 \pi}{L} P_{\xi} x-\sqrt{\frac{1}{g}} \sum_{n \neq 0} \sin \left[\frac{\pi n}{L} x\right] \frac{\alpha_{\xi}(n)}{n} e^{-i \frac{\pi n}{L} u t}
$$

with $\left[a_{\xi}(n), a_{\xi}\left(n^{\prime}\right)\right]=n \delta_{n+n^{\prime}, 0}, k_{n}=\pi n / L$; the 0-mode operator of the field $\xi$, $P_{\xi}$, has the eigenvalues

$$
P_{\xi}=\left[\frac{\varphi}{4 \pi}+\frac{m}{2}\right] \quad ; \quad\left(m=n_{1}-n_{2}\right)
$$

Similarly, one obtains

$$
X(x, t)=q_{X}+i \sqrt{\frac{1}{g}} \sum_{n \in Z} \cos \left[\frac{\pi}{L}\left(n+\frac{1}{2}\right) x\right] \frac{\alpha_{X}(n)}{n+\frac{1}{2}} e^{-i \frac{\pi}{L}\left(n+\frac{1}{2}\right) u t}
$$

with $\left[\alpha_{X}(n), \alpha_{X}\left(n^{\prime}\right)\right]=n \delta_{n+n^{\prime}, 0}, K_{n}=\frac{\pi}{L}\left(n+\frac{1}{2}\right), q_{X}=\sqrt{\frac{g}{2}} \pi\left(n_{1}+n_{2}\right)$.

As for the rf-SQUID, the strongly coupled fixed point is described in terms of the (now two!) dual fields $\Theta_{X}(x, t)$ and $\Theta_{\xi}(x, t)$, whose mode expansion is given by

$$
\Theta_{X}(x, t)=-2 \pi \tilde{P}_{X}-2 \sqrt{g} \sum_{n \in Z} \sin \left[\frac{\pi}{L}\left(n+\frac{1}{2}\right) x\right] \frac{\alpha_{X}(n)}{n+\frac{1}{2}} e^{-i \frac{\pi}{L}\left(n+\frac{1}{2}\right) u t}
$$


where $P_{X}$ is the canonical conjugate operator of $q_{X}$, and

$$
\Theta_{\xi}(x, t)=\theta_{0}+\frac{2 \pi}{L} g P_{\xi} u t+i 2 \sqrt{g} \sum_{n \neq 0} \cos \left[\frac{\pi n}{L} x\right] \frac{\alpha_{\xi}(n)}{n} e^{-i \frac{n \pi}{L} u t} .
$$

Following the same steps used in subsection (2.2), one has that

$$
H_{D}\left[\Theta_{X}, \Theta_{\xi}\right]=\frac{2 \pi v_{\mathrm{pl}} g}{L}\left(P_{\xi}\right)^{2}+\sum_{n \neq 0}\left[\alpha_{X}(-n) \alpha_{X}(n-1)+\alpha_{\xi}(-n) \alpha_{\xi}(n)\right]
$$

from which it is straightforward to compute the partition function at the Dirichlet fixed point:

$$
\mathbf{Z}_{D}=\operatorname{Tr}\left[e^{-\beta H_{D}\left[\Theta_{X}, \Theta_{\xi}\right]}\right]=\prod_{n=0}^{\infty}\left[\left(\frac{1}{1-q^{n+\frac{1}{2}}}\right)\left(\frac{1}{1-q^{n+1}}\right)\right] \sum_{k \in Z} \exp \left[-\beta \frac{\pi v_{\mathrm{pl}} g}{2 L}\left(k-\frac{\varphi}{2 \pi}\right)^{2}\right]
$$

Eq.(95) shows that, for $\varphi \neq 2 k \pi+\pi$, with $k$ integer, the ground state is characterized by a nonzero value of $P_{\xi}$, proportional to $[\varphi]$. Tunneling events from this ground state to the nearest (in energy) ground states are suppressed by the exponential factor $\exp \left[-\beta \pi v_{\mathrm{pl}} g / 2 L\right]$.

Taking the logarithmic derivative of Eq.(95) one easily derives the Josephson current, which is given by

$$
I[\varphi]=-e^{*} \lim _{\beta \rightarrow \infty} \frac{1}{\beta} \frac{\partial \ln \mathbf{Z}_{D}[\varphi]}{\partial \varphi}=\frac{e^{*} g v_{\mathrm{pl}}}{4 \pi L}\{\varphi-[\varphi]\}
$$

From Eq.(95), one sees that the degeneracy among the minima of the Josephson energy is removed by the magnetic energy $E_{m}^{(0)}=\frac{2 g \pi v_{\mathrm{pl}}}{L}\left(-\frac{\varphi}{4 \pi}+\frac{m}{2}\right)^{2}$. Since $E_{m}^{(0)}=E_{m+1}^{(0)}$, for $\varphi=\pi+2 \pi m$ also a dc-SQUID may operate as a qubit between these two levels. When the couplings are large, but finite, phase slips in the plasmon field (instantons) will induce a renormalization of the physical parameters, as we shall show in the following subsection.

\subsection{Instantons and boundary perturbations at the Dirichlet fixed point}

In order to construct the leading boundary perturbations at the strongly coupled fixed point, one may use again the DEBC-method [19], applied to the 
primary bulk operators involving the dual fields $\Theta_{X}, \Theta_{\xi}$. A generic, imaginary time, bulk operator at the strongly coupled fixed point may be written as $V_{\alpha, \beta}(i \tau)=: \exp \left\{i\left[\alpha \Theta_{X}(i \tau)+\beta \Theta_{\xi}(i \tau)\right]\right\}$, where the imaginary time boundary fields are given by

$$
\Theta_{X}(i \tau)=\Theta_{X}(L, i \tau)=-2 \pi \tilde{P}_{X}-2 \sqrt{g} \sum_{n \in Z}(-1)^{n} \frac{\alpha_{X}(n)}{n+\frac{1}{2}} e^{-\frac{\pi}{L}\left(n+\frac{1}{2}\right) u \tau}
$$

and by

$$
\Theta_{\xi}(i \tau)=\Theta_{\xi}(L, i \tau)=\theta_{0}+\frac{2 \pi}{L} 2 g P_{\xi} i u \tau+i 2 \sqrt{g} \sum_{n \neq 0}(-1)^{n} \frac{\alpha_{\xi}(n)}{n} e^{-\frac{n \pi}{L} u \tau}
$$

From the commutation rules between the zero modes of the two fields, one obtains

$$
\left[q_{X}, V_{\alpha, \beta}(i \tau)\right]=2 \pi \alpha V_{\alpha, \beta}(i \tau) \quad ; \quad\left[P_{\xi}, V_{\alpha, \beta}(i \tau)\right]=2 \pi \beta V_{\alpha, \beta}(i \tau)
$$

Thus, $V_{\alpha, \beta}(i \tau)$ changes the eigenvalue of $q_{X}$ by $2 \pi \alpha$ and the eigenvalue of $P_{\xi}$ by $2 \pi \beta$. Using Eqs.(97,98,99), one may write the leading boundary operators as

$$
\begin{aligned}
& \tilde{V}_{a, b}(i \tau)=: \exp \left\{\frac{i}{2}\left[a \Theta_{X}(i \tau)+b \Theta_{\xi}(i \tau)\right]\right\} \quad ; \quad a, b= \pm 1 \\
& \tilde{V}_{X, \pm}(i \tau)=: \exp \left\{ \pm i \Theta_{X}(i \tau)\right\}: ; \quad \tilde{V}_{\xi, \pm}(i \tau)=: \exp \left\{ \pm i \Theta_{\xi}(i \tau)\right\}
\end{aligned}
$$

$\tilde{V}_{1,1}(i \tau)$ and $\tilde{V}_{-1,-1}(i \tau)\left(\tilde{V}_{1,-1}(i \tau)\right.$ and $\left.\tilde{V}_{-1,1}(i \tau)\right)$ change by $\pm 2 \pi$ the phase at junction 1 (2), while they leave the phase at junction 2 (1) unchanged and thus they describe phase slip operators at junction 1 (2). Similarly, $\tilde{V}_{X, \pm}(i \tau)$ $\left(\tilde{V}_{\xi, \pm}(i \tau)\right)$ change the eigenvalue of the overall (relative) phase $X(L)(\xi(L))$ by $\pm 2 \pi$ leaving the eigenvalue of $\xi(L)(X(L))$ unchanged: thus, $\tilde{V}_{X, \pm}(i \tau)$ do not affect the Josephson current across the ring, while $\tilde{V}_{\xi, \pm}(i \tau)$ provide corrections to the Josephson current when $\varphi=2 k \pi+\pi$. In the following we shall refer to $\tilde{V}_{X, \pm}(i \tau)\left(\tilde{V}_{\xi, \pm}(i \tau)\right)$ as $q^{-}(\pi-)$ Instanton operators.

The leading boundary perturbation at the strongly coupled fixed point is given by an arbitrary linear combinations of the operators listed above, as

$$
\tilde{H}_{d c, J}=Y_{1}\left[\tilde{V}_{1,1}(i \tau)+\tilde{V}_{-1,-1}(i \tau)\right]+Y_{2}\left[\tilde{V}_{1,-1}(i \tau)+\tilde{V}_{-1,1}(i \tau)\right]
$$




$$
+Y_{X}\left[\tilde{V}_{X,+}(i \tau):+\tilde{V}_{X,-}(i \tau)\right]+Y_{\xi}\left[\tilde{V}_{\xi,+}(i \tau):+\tilde{V}_{\xi,-}(i \tau)\right]
$$

From the mode expansion of the dual fields, it is straightforwad to compute the (low-temperature) correlators among the boundary vertex operators appearing in Eq.(102). For $|\varphi|<\pi$, for instance, one has that

$$
\begin{aligned}
& \left\langle\mathbf{T}_{\tau}\left[\tilde{V}_{1,1}(i \tau) \tilde{V}_{-1,-1}\left(i \tau^{\prime}\right)\right]\right\rangle=\left\langle\mathbf{T}_{\tau}\left[\tilde{V}_{-1,1}(i \tau) \tilde{V}_{1,-1}\left(i \tau^{\prime}\right)\right]\right\rangle=\frac{e^{\frac{ \pm g v_{\mathrm{pl}} \varphi}{4 L}\left(\tau-\tau^{\prime}\right)}}{\left|\sinh \left[\frac{\pi v_{\mathrm{pl}}}{4 L}\left(\tau-\tau^{\prime}\right)\right]\right|^{2 g}},(10) \\
& \left\langle\mathbf{T}_{\tau}\left[\tilde{V}_{+, X}(i \tau) \tilde{V}_{-, X}\left(i \tau^{\prime}\right)\right]\right\rangle=\frac{\left|\cosh \left[\frac{\pi v_{\mathrm{pl}}}{4 L}\left(\tau-\tau^{\prime}\right)\right]\right|^{4 g}}{\left|\sinh \left[\frac{\pi v_{\mathrm{p}}}{4 L}\left(\tau-\tau^{\prime}\right)\right]\right|^{4 g}}
\end{aligned}
$$

and

$$
\left\langle\mathbf{T}_{\tau}\left[\tilde{V}_{+, \xi}(i \tau) \tilde{V}_{-, \xi}\left(i \tau^{\prime}\right)\right]\right\rangle=\frac{e^{ \pm \frac{g v_{\mathrm{p}} \varphi}{L}\left(\tau-\tau^{\prime}\right)}}{\left|2 \sinh \left[\frac{\pi v_{\mathrm{pl}}}{2 L}\left(\tau-\tau^{\prime}\right)\right]\right|^{4 g}}
$$

Eqs. 103]104 105) imply that the fugacity of the q- and $\pi$-instantons and of the phase slips at the junctions scale with the SQUID size as

$$
Y_{X(\xi)}(L)=Y_{X(\xi)}\left(v_{\mathrm{pl}} T_{x}\right)\left(\frac{L}{v_{\mathrm{p} 1} T_{x}}\right)^{1-2 g} ; Y_{1(2)}(L)=Y_{1(2)}\left(v_{\mathrm{pl}} T_{x}\right)\left(\frac{L}{v_{\mathrm{p} 1} T_{x}}\right)^{1-g}
$$

The "instanton size" $T_{x}$ in Eq.(106) is estimated to be of the order of $\sqrt{g L /\left[\pi v_{\mathrm{pl}}\left(E_{J, 1}+E_{J, 2}\right)\right]}$ [7].

Eq.(106) shows that, for $g<1$, phase slips at the two junctions provide the most relevant perturbation to the Dirichlet fixed point, rendering the strongly coupled fixed point not infrared stable. For finite $\beta, L$ and for $\varphi \neq 2 \pi k+$ $\pi$, however, the phase slips are always suppressed by the magnetic energy associated to the eigenvalue of $P_{\xi}$. At variance, when $g>1, \pi$-instantons are an irrelevant perturbation which, for $L<L_{*, 1}, L_{*, 2}$ (i.e., of the healing lengths associated to the two junctions), only smooths down the edges of the sawtooth shape of the Josephson current: for $L \rightarrow \infty$, the ground state exhibits a discrete Z-symmetry. 


\section{Transport in SQUID devices}

To probe the different regimes attainable by SQUID devices, it is most useful to look at their dc-conductance. To get conduction of current across a SQUID device, one should be able to connect it to two leads, enabling to apply a biasing voltage $V$. This can be achieved easily for a dc-SQUID while, for the purposes of this section, it is most convenient to regard the rf-SQUID as an inhomogeneous chain of Josephson junctions connected to two bulk superconducting leads at fixed phase difference $\varphi$ and at finite biasing voltage $V$; the chain is made by junctions of equal strength with a weak link of nominal strength $E_{J}$ located at its center. For $V=0$, this inhomogeneous chain mimicks the response of an rf-SQUID to an external magnetic flux $\varphi$ [21]. In this section we study the trasport properties of both devices, evidencing the different current (normal and Josephson) response at weak and at strong coupling. Our boundary field theory approach well reproduces the known results of Refs. [7,9] and, for a dc-SQUID, evidences interesting interference effects between the current flowing through the two junctions.

\subsection{The Josephson junction chain at the weakly coupled fixed point}

The relevant dynamics of a Josephson junction chain with a weak link connected to two bulk superconductors at fixed phase difference $\varphi$ has been altready analyzed in Refs. [35,21]. The properties of this device may be described by the bosonic field $\Phi(x)=\frac{1}{\sqrt{2}}\left[\Phi_{R}(x)-\Phi_{L}(-x)\right]$, where $\Phi_{R}(x)$ and $\Phi_{L}(x)$ are the fields describing the phase of each junction in the right and the left halfchain, respectively. The current operator across the junction is then given by

$j(t)=-\frac{g e^{*} v_{\mathrm{pl}}}{\sqrt{2} \pi} \frac{\partial \Phi(L, t)}{\partial x}$. Energy conservation provides the dynamical boundary conditions for $\Phi(x, t)$ at the outer boundary, which is given by

$$
\frac{v_{\mathrm{pl}} g}{\pi} \frac{\partial \Phi(L, t)}{\partial x}+\sqrt{2} \bar{E}_{J} \sin [\sqrt{2} \Phi(L, t)+\varphi]=0
$$

using Eq.(107), the current operator may be written as

$$
j(t)=e^{*} \bar{E}_{J} \sin [\sqrt{2} \Phi(t)+\varphi] \quad ; \quad(\Phi(t)=\Phi(L, t)) \quad .
$$

At the weakly coupled fixed point $\left(E_{J}=0\right), \Phi(x, t)$ satisfies the Neumann boundary conditions at the outer boundary; namely, $\frac{\partial \Phi(L, t)}{\partial x}=0$.

In order to apply a voltage $V$ to the device, one may bias at voltage $V / 2$ and $-V / 2$ each one of the two leads. In the small $\bar{E}_{J}$-limit, it is safe to assume that 
each chain is at equilibrium with its own lead, and that the two half chains are disconnected. Accordingly, the voltage bias $V$ is introduced by adding to the total Hamiltonian the term

$$
H_{V}=-\frac{e^{*} V g}{\pi v_{\mathrm{pl}}} \int_{0}^{L} d x \frac{\partial \Phi}{\partial t}
$$

which can be accounted for by a mere shifting of the field $\Phi$, as

$$
\Phi(x, t) \rightarrow \Phi(x, t)-e^{*} V t
$$

As a result of Eq.(110), the Josephson current acquires an explicit dependence on time and, to the first order in $\bar{E}_{J}$, reads

$$
I_{J}[\varphi, t]=e^{*} \bar{E}_{J} \sin \left[\varphi+e^{*} V t\right] .
$$

Thus, in addition to the Josephson current, there is a normal time-independent dc-current which, as it will be shown in the following, becomes the leading contribution for a pertinent choice of the infrared cutoff leading to the celebrated Kane-Fisher formula [9].

Since the normal current is independent of time, it may be computed using the imaginary time formalism. From Eq.(110), one obtains

$$
j(i \tau)=e^{*} \bar{E}_{J}: \sin \left[\sqrt{2} \Phi(i \tau)+\varphi+e^{*} V i \tau\right]:,
$$

with

$$
\Phi(i \tau)=-\sqrt{\frac{2}{g}} \sum_{n}(-1)^{n} \frac{\alpha(n)}{n+\frac{1}{2}} e^{-\frac{\pi}{L}\left(n+\frac{1}{2}\right) v_{\mathrm{p} 1} \tau} .
$$

From Eqs.(112,113), it follows that the leading contribution to the normal current appears only at the second order in $\bar{E}_{J}$, since

$$
\begin{aligned}
& I \approx \frac{e^{*}}{4 i}\left(\bar{E}_{J}\right)^{2} \int_{0}^{\beta} d \tau^{\prime}\left\langle\mathbf{T}_{\tau}\left[: \exp \left[\sqrt{2} \Phi(i \tau)+\varphi+i e^{*} V \tau\right]:: \exp \left[-\sqrt{2} \Phi\left(i \tau^{\prime}\right)-\varphi-i e^{*} V \tau^{\prime}\right]:\right]\right\rangle \\
& -\frac{e^{*}}{4 i}\left(\bar{E}_{J}\right)^{2} \int_{0}^{\beta} d \tau^{\prime}\left\langle\mathbf{T}_{\tau}\left[: \exp \left[-\sqrt{2} \Phi(i \tau)-\varphi-i e^{*} V \tau\right]:: \exp \left[\sqrt{2} \Phi\left(i \tau^{\prime}\right)+\varphi+i e^{*} V \tau^{\prime}\right]:\right]\right\rangle
\end{aligned}
$$




$$
=e^{*}\left(\bar{E}_{J}\right)^{2} \frac{2 L}{\pi v_{\mathrm{pl}}} \int_{0}^{\infty} d x\left[\frac{\sinh [a x]}{2^{\frac{2}{g}} \sinh ^{\frac{2}{g}}(x)}\right]=\frac{2 e^{*} \pi\left(\bar{E}_{J}\right)^{2} L}{\pi v_{\mathrm{pl}}} \mathbf{I}[a]
$$

with

$$
a=\frac{2 L e^{*} V}{\pi v_{\mathrm{pl}}} .
$$

and 36

$$
\mathbf{I}[a]=\int_{0}^{\infty} d x \frac{\sinh [a x]}{[2 \sinh (x)]^{\frac{2}{g}}}=\frac{\Gamma\left[1-\frac{2}{g}\right]}{4}\left\{\frac{\Gamma\left[-\frac{a}{2}+\frac{1}{g}\right]}{\Gamma\left[1-\frac{a}{2}-\frac{1}{g}\right]}-\frac{\Gamma\left[\frac{a}{2}+\frac{1}{g}\right]}{\Gamma\left[1+\frac{a}{2}-\frac{1}{g}\right]}\right\}
$$

From Eq.(114), one sees that the dc-current depends crucially on the ratio $a$ between the energy window defined by the applied voltage, $e^{*} V$, and the level spacing characteristic of the device, $\pi v_{\mathrm{pl}} /(2 L)$. In fact, if $e^{*} V<\pi v_{\mathrm{pl}} / L$, one may expand Eq.(114) to first order in $a$, getting

$$
I=\frac{e^{*}\left(\bar{E}_{J}\right)^{2} L \Gamma\left[1-\frac{2}{g}\right]}{2 v_{\mathrm{pl}}} B V
$$

with

$$
B=\left.\frac{\partial}{\partial z}\left\{\frac{\Gamma\left[-\frac{z}{2}+\frac{1}{g}\right]}{\Gamma\left[1-\frac{z}{2}-\frac{1}{g}\right]}-\frac{\Gamma\left[\frac{z}{2}+\frac{1}{g}\right]}{\Gamma\left[1+\frac{z}{2}-\frac{1}{g}\right]}\right\}\right|_{z=0}
$$

which yields the (normal) dc-linear response, to an applied voltage $V$, of a junction with renormalized strength $\bar{E}_{J}=\left(\frac{2 \pi a}{L}\right)^{\frac{1}{g}}$; this is expected, since, in this limit, $\pi v_{\mathrm{pl}} / L$ provides the infrared cutoff. If, instead, $v_{\mathrm{pl}} / L \ll e^{*} V$, one may compute the current in a large- $a$ expansion, using the Stirling formula, $\Gamma[z] \approx \sqrt{2 \pi} e^{-z} z^{z-\frac{1}{2}}$, which yields

$$
I=\frac{e^{*}\left(\bar{E}_{J}\right)^{2} L}{\pi v_{\mathrm{pl}}} \sin \left[\frac{\pi}{g}\right] \Gamma\left[1-\frac{2}{g}\right]\left[\frac{2 L e^{*} V}{\pi v_{\mathrm{pl}}}\right]^{\frac{2}{g}-1} .
$$

Eq.(119) is the celebrated Kane-Fisher formula for the current across a constriction in a spinless Luttinger liquid. Our result is, after all, not surpris- 
ing, since the wires connecting the junctions have been regarded as onedimensional spinless Luttinger liquids, and the junctions as boundary interactions. It should be noticed, however, that the normal current is proportional to $\left(e^{*} V\right)^{\frac{2}{g}-1}$ since $e^{*} V$ is the pertinent infrared cutoff when an external bias voltage is applied to the device. At variance, in the computation of the Josephson current, the pertinent cutoff is provided by the plasmon's level spacing $s=2 \pi v_{\mathrm{pl}} / L$. Thus, in the limit where $s \ll e^{*} V$, our analysis shows that the leading contribution to the total current across the junction is given by the normal current.

\subsection{The Josephson junction chain at the strongly coupled fixed point}

In this section, the current across the Josephson junction chain is computed at zero phase difference $\varphi$ as $\bar{E}_{J} /(\pi u / L) \rightarrow \infty$. Here, it is most convenient to resort to the dual formulation, based on the dual field $\Theta(x, t)$. The total Hamiltonian (including the leading boundary perturbation) is given by

$$
H[\Theta]=\frac{1}{4 \pi(2 g)} \int_{0}^{L} d x\left[\frac{1}{u}\left(\frac{\partial \Theta}{\partial t}\right)^{2}+u\left(\frac{\partial \Theta}{\partial x}\right)^{2}\right]-Y\left[: e^{\frac{i}{\sqrt{2}} \Theta(L)}:+: e^{\frac{i}{\sqrt{2}} \Theta(L)}:\right]
$$

where the dual field $\Theta(x, t)$ obeys to Neumann boundary conditions at $x=L$. Thus, the mode expansion of the field $\Theta(i \tau)=\Theta(L, i \tau)$ is given by

$$
\Theta(i \tau)=\Theta(L, i \tau)=\theta_{0}+i \frac{2 \pi}{L} 2 g P u \tau+i 2 \sqrt{g} \sum_{n \neq 0}(-1)^{n} \frac{\alpha(n)}{n} e^{-\frac{\pi n}{L} u \tau} .
$$

Since at the strongly coupled fixed point, the two half chains cannot be regarded as isolated from each other, one must apply a voltage $V$ by biasing the right-handed chiral mode of $\Phi(x, t)$, with respect to the left-handed ones. This corresponds to adding to $H[\Theta]$ a voltage-dependent term given by

$$
\tilde{H}_{V}=-\frac{e^{*} V}{2 \pi} \int_{0}^{L} d x \frac{\partial \Phi}{\partial x}=-\frac{e^{*} V}{2 \pi v_{\mathrm{pl}}} \int_{0}^{L} d x \frac{\partial \Theta}{\partial t}
$$

The imaginary time current operator is

$$
j(i \tau)=\left.\frac{\delta S_{E}[\Theta ; V, a]}{\delta a(\tau)}\right|_{a=0}
$$


where $S_{E}[\Theta ; V, a]$ is the Euclidean action with a source term for the current, given by

$$
\begin{aligned}
& S_{E}[\Theta ; V, a]=\frac{1}{4 \pi(2 g)} \int_{0}^{\beta} d \tau \int_{0}^{L} d x\left[\frac{1}{v_{\mathrm{pl}}}\left(\frac{\partial \Theta(x, i \tau)}{\partial \tau}\right)^{2}+v_{\mathrm{pl}}\left(\frac{\partial \Theta(x, i \tau)}{\partial x}\right)^{2}\right] \\
& -2 Y \int_{0}^{\beta} d \tau: \cos \left(\frac{\Theta(i \tau)}{\sqrt{2}}\right): \\
& -i \frac{e^{*} V}{2 \pi v_{\mathrm{pl}}} \int_{0}^{\beta} d \tau \int_{0}^{L} d x \frac{\partial \Theta(x, i \tau)}{\partial \tau}-\frac{i e^{*}}{2 \pi L} \int_{0}^{\beta} d \tau \int_{0}^{L} d x a(\tau) \frac{\partial \Theta(i \tau)}{\partial \tau}
\end{aligned}
$$

By shifting the field $\Theta$ according to

$$
\frac{\Theta(x, i \tau)}{\sqrt{2}} \rightarrow \frac{\Theta(x, i \tau)}{\sqrt{2}}-2 i g e^{*} V \tau-i \frac{e^{*} g v_{\mathrm{pl}}}{L} A(\tau)
$$

with $A^{\prime}(\tau)=a(\tau)$, one finds that the current is given by

$$
\tilde{j}(\tau)=\left.\frac{\delta \tilde{S}_{E}[\Theta ; V, a]}{\delta a(\tau)}\right|_{a=0}=\frac{g\left(e^{*}\right)^{2}}{\pi} V+4 Y \frac{e^{*} g v_{\mathrm{pl}}}{L} \sin \left(\frac{\Theta(i \tau)}{\sqrt{2}}-i e^{*} V \tau\right):
$$

From Eq.(126) one sees that, apart from the constant term $\frac{g\left(e^{*}\right)^{2}}{\pi} V$, the current may be computed as in the previous section, provided that one substitutes $4 / \mathrm{g}$ with $g$ and $\bar{E}_{J}$ with $Y$. Thus, to the second order in $Y$ one obtains

$$
I=\frac{g\left(e^{*}\right)^{2}}{\pi} V+\frac{2 e^{*} Y^{2} L}{\pi} \sin [g \pi] \Gamma[1-2 g]\left[\frac{2 L e^{*} V}{\pi v_{\mathrm{pl}}}\right]^{2 g-1} .
$$

As it happens also at the weakly coupled fixed point, the celebrated power-law dependence of $I$ on the applied voltage $V$ emerges when $2 L e^{*} V /\left(\pi v_{\mathrm{pl}}\right) \gg 1$.

\subsection{The dc-SQUID at the weakly coupled fixed point}

For a dc-SQUID, it is most convenient to represent the leads as two quantum wires with the same Luttinger parameters $g$ and $v_{\mathrm{pl}}$, with plasmon fields respectively given by $\Phi_{L}$ and $\Phi_{R}$. The two leads are assumed to be connected 
to the dc-SQUID with smooth junctions, that is, three-wire junctions with no backscattering in any arm. Thus, one should describe the SQUID's arm with four fields, rather than with two; namely $\Phi_{u, L}, \Phi_{u, R}$ for the left-upper and for the right-upper arm of the ring, $\Phi_{d, L}, \Phi_{d, R}$ for left-lower and for the rightlower arm of the ring, respectively. Since the connections between leads and SQUID's arm are assumed to be ideal, they are described by the strongly coupled fixed point of the three quantum wire "Y"-junction studied in Ref.[19], with no concatenated flux. Thus, by centering the Y-junction at $x=0$, the boundary conditions are given by

$$
\left.\frac{\partial}{\partial x}\left[\Phi_{a}(x, t)+\Phi_{u, a}(x, t)+\Phi_{d, a}(x, t)\right]\right|_{x=0}=0
$$

which embodies the current conservation at the connection, and by

$$
-2 \Phi_{a}(0, t)+\Phi_{u, L}(0, t)+\Phi_{d, a}(0, t)=\Phi_{u, a}(0, t)-\Phi_{d, a}(0, t)=0,
$$

with $a=L, R$.

The relevant fields for the Josephson junction dynamics are given by the "oddparity" combinations

$$
\Phi_{u / d}(x, t)=\frac{1}{\sqrt{2}}\left[\Phi_{u / d, R}(x, t)-\Phi_{u / d, L}(x, t)\right] ; \quad \Phi(x, t)=\frac{1}{\sqrt{2}}\left[\Phi_{R}(x, t)-\Phi_{L}(x, t)\right]
$$

which are related to the fields $\xi(x, t), X(x, t)$ introduced in section (3) by

$$
\Phi_{u}(x, t)=\frac{1}{2}[X(x, t)+\xi(x, t)] \quad ; \quad \Phi_{d}(x, t)=\frac{1}{2}[X(-x, t)-\xi(-x, t)]
$$

As one sees from Eqs.(128, 129), introducing the contacts does not affect at all the boundary condition of $\xi(x, t)$ at the inner boundary $(x=0)$. The boundary conditions for $X(x, t)$, instead, explicitly depend on the applied voltage bias, whose effect may be accounted for by including in the total Hamiltonian a pertinent "source" term.

At the weakly coupled fixed point, biasing the left-hand lead at a dc-voltage $V / 2$ and the right-hand lead at a voltage $-V / 2$, corresponds to adding to the SQUID's Hamiltonian a voltage dependent term given by

$$
H_{V}=-\frac{g \sqrt{2} e^{*} V}{4 \pi} \int_{-L}^{L} d x\left[\frac{\partial \Phi_{R}(x)}{\partial t}-\frac{\partial \Phi_{L}(x)}{\partial t}\right]=-\frac{g e^{*} V}{2 \pi} \int_{0}^{L} d x \frac{\partial X(x)}{\partial t}
$$


which is equivalent to a shift of $\Phi(x, t)$, which is linear in time, namely, the field $\Phi(x, t)$ should be replaced by $\Phi(x, t)-e^{*} V t$. From Eqs.(128]129]132) one gets that also $\Phi_{u / d}(x, t)$ must be shifted as

$$
\Phi_{u / d}(x, t) \rightarrow \Phi_{u / d}(x, t)-\frac{e^{*} V}{2} t
$$

As a consequence, one sees that $X(x, t)$ takes the additional contribution, given by

$$
X(x, t) \rightarrow X(x, t)-e^{*} V t
$$

which introduces in the boundary interaction Hamiltonian an explicit dependence on $\tau$, given by

$$
\begin{aligned}
& H_{d c, J} \rightarrow H_{d c, J}(i \tau)=-\bar{E}_{J, 1} \cos \left[X(i \tau)+\xi(i \tau)+\frac{\varphi}{2}+i e^{*} V \tau\right] \\
& -\bar{E}_{J, 2} \cos \left[X(i \tau)-\xi(i \tau)-\frac{\varphi}{2}+i e^{*} V \tau\right] ;
\end{aligned}
$$

at finite $V$, the current operators across the two junctions are then given by

$$
j_{1}(i \tau)=e^{*} \bar{E}_{J, 1} \sin \left[X(i \tau)+\xi(i \tau)+\frac{\varphi}{2}+i e^{*} V \tau\right]
$$

and

$$
j_{2}(i \tau)=e^{*} \bar{E}_{J, 2} \sin \left[X(i \tau)-\xi(i \tau)+\frac{\varphi}{2}-i e^{*} V \tau\right]
$$

As in the previous section, the current across the two junctions is computed from a perturbative expansion in $\bar{E}_{J, 1}, \bar{E}_{J, 2}$. One obtains the following results

- Current across the junction 1: $I_{1}$

$$
I_{1}=I_{1,1}+I_{1,2}
$$


$I_{1,1}$ is given by

$$
\begin{aligned}
& I_{1,1}=e^{*}\left(\bar{E}_{J, 1}\right)^{2} \int_{0}^{\beta} d \tau^{\prime}\left\langle\mathbf { T } _ { \tau } \left\{: \sin \left[X(i \tau)+\xi(i \tau)+\frac{\varphi}{2}+i e^{*} V \tau\right]: \times\right.\right. \\
& \left.\left.: \cos \left[X\left(i \tau^{\prime}\right)+\xi\left(i \tau^{\prime}\right)+\frac{\varphi}{2}+i e^{*} V \tau^{\prime}\right]:\right\}\right\rangle=\frac{e^{*}}{2}\left(\bar{E}_{J, 1}\right)^{2} \int_{0}^{\infty} d \tau\left\{\frac{\sinh \left[e^{*} V \tau\right]}{\left[2 \sinh \left(\frac{v_{\mathrm{p}} \pi}{2 L} \tau\right)\right]^{\frac{2}{g}}}\right\},
\end{aligned}
$$

which, for $4 L e^{*} V /\left(v_{\mathrm{pl}} \pi\right) \gg 1$ yields

$$
\begin{aligned}
& I_{1,1} \approx \frac{2 e^{*}\left(\bar{E}_{J, 1}\right)^{2} L}{\pi v_{\mathrm{pl}}} \sin \left[\frac{\pi}{g}\right] \Gamma\left[1-\frac{2}{g}\right]\left[\frac{4 L e^{*} V}{\pi v_{\mathrm{pl}}}\right]^{\frac{2}{g}-1} \\
& I_{1,2}=e^{*} \bar{E}_{J, 1} \bar{E}_{J, 2} \int_{0}^{\beta} d \tau^{\prime}\left\langle\mathbf { T } _ { \tau } \left\{: \sin \left[X(i \tau)+\xi(i \tau)+\frac{\varphi}{2}+e^{*} V \tau\right]: \times\right.\right. \\
& \left.\left.: \cos \left[X\left(i \tau^{\prime}\right)-\xi\left(i \tau^{\prime}\right)-\frac{\varphi}{2}+e^{*} V \tau^{\prime}\right]:\right\}\right\rangle \\
& \approx e^{*} \bar{E}_{J, 1} \bar{E}_{J, 2} \int_{0}^{\infty} d \tau\left\{\frac{e^{e^{*} V \tau+i \varphi}}{\left[\cosh \left(\frac{\pi v_{\mathrm{pl}}}{4 L} \tau\right)\right]^{\frac{2}{g}}}-\frac{e^{-e^{*} V \tau-i \varphi}}{\left[\cosh \left(\frac{\pi v_{\mathrm{pl}}}{4 L}\right)\right]^{\frac{2}{g}}}\right\} \\
& =\frac{4 e^{*} \bar{E}_{1} \bar{E}_{J, 2} L}{\pi v_{\mathrm{pl}}}\left\{e^{i \varphi} \mathbf{J}[a]-e^{-i \varphi} \mathbf{J}[-a]\right\}
\end{aligned}
$$

with

$$
\mathbf{J}[a]=\int_{0}^{\infty} d x \frac{e^{a x}}{\cosh ^{\frac{2}{g}}(x)}
$$

where $a=4 e^{*} V L /\left(v_{\mathrm{pl}} \pi\right)$ and

$$
\mathbf{J}[a]=2^{\frac{2}{g}-1} e^{-i \pi\left(\frac{a}{2}+\frac{1}{g}\right)} B\left[-1 ;-\left(\frac{a}{2}-\frac{1}{g}\right) ; 1-\frac{2}{g}\right],
$$

where the incomplete - Beta function is defined as $B[z, \alpha, \beta]=\int_{0}^{z} d t t^{\alpha-1}(1-$ t) $)^{\beta-1}$

For large $a$ and $1-2 / g>0$, one may consider the asymptotic expansion of the incomplete- Beta function [36],

$$
B\left[-1 ;-\left(\frac{a}{2}+\frac{1}{g}\right) ; 1-\frac{2}{g}\right] \approx i \exp \left[-i \frac{\pi}{2} a-i \frac{\pi}{2}\left(\frac{2}{g}-1\right)\right] a^{\frac{2}{g}-1} \Gamma\left[\frac{2}{g}-1\right],
$$

from which

$$
I_{1,2} \approx \frac{2 e^{*} \bar{E}_{J, 1} \bar{E}_{J, 2} L}{\pi v_{\mathrm{pl}}} \Gamma\left[1-\frac{2}{g}\right]\left\{\cos (\varphi) \cos \left[\frac{2 e^{*} V L}{v_{\mathrm{pl}}}+\frac{\pi}{g}\right]\right.
$$




$$
\left.+\sin (\varphi) \sin \left[\frac{2 e^{*} V L}{v_{\mathrm{pl}}}+\frac{\pi}{g}\right]\right\}\left[\frac{4 L e^{*} V}{\pi v_{\mathrm{pl}}}\right]^{\frac{2}{g}-1}
$$

- Current across the junction 2: $I_{2}$

$$
I_{2}=I_{2,1}+I_{2,2}
$$

The two contributions are computed below:

$$
\begin{aligned}
& I_{2,2}=e^{*}\left(\bar{E}_{J, 2}\right)^{2} \int_{0}^{\beta} d \tau^{\prime}\left\langle\mathbf { T } _ { \tau } \left\{: \sin \left[X(i \tau)+\xi(i \tau)+\frac{\varphi}{2}-i e^{*} V \tau\right]: \times\right.\right. \\
& \left.\left.: \cos \left[X\left(i \tau^{\prime}\right)+\xi\left(i \tau^{\prime}\right)+\frac{\varphi}{2}-i e^{*} V \tau^{\prime}\right]:\right\}\right\rangle \approx \frac{2 e^{*}\left(\bar{E}_{J, 2}\right)^{2} L}{\pi v_{\mathrm{pl}}} \sin \left[\frac{\pi}{g}\right] \Gamma\left[1-\frac{2}{g}\right]\left[\frac{4 L e^{*} V}{\pi v_{\mathrm{pl}}}\right]^{\frac{2}{g}-1} \\
& I_{2,1}=e^{*} \bar{E}_{J, 1} \bar{E}_{J, 2} \int_{0}^{\beta} d \tau^{\prime}\left\langle\mathbf { T } _ { \tau } \left\{: \sin \left[X(i \tau)+\xi(i \tau)-\frac{\varphi}{2}+e^{*} V \tau\right]: \times\right.\right. \\
& \left.\left.: \cos \left[X\left(i \tau^{\prime}\right)-\xi\left(i \tau^{\prime}\right)+\frac{\varphi}{2}+e^{*} V \tau^{\prime}\right]:\right\}\right\rangle \\
& \approx \frac{4 e^{*} \bar{E}_{1} \bar{E}_{J, 2} L}{\pi v_{\mathrm{pl}}} \Gamma\left[1-\frac{2}{g}\right]\left\{\cos (\varphi) \cos \left[\frac{2 e^{*} V L}{v_{\mathrm{pl}}}+\frac{\pi}{g}\right]\right. \\
& \left.+\sin (\varphi) \sin \left[\frac{2 e^{*} V L}{v_{\mathrm{pl}}}+\frac{\pi}{g}\right]\right\}\left[\frac{4 L e^{*} V}{\pi v_{\mathrm{pl}}}\right]^{\frac{2}{g}-1} \cdot
\end{aligned}
$$

The net transport current across the dc-SQUID is obtained as the sum of the currents across the two junctions, that is

$$
\begin{aligned}
& I=I_{1}+I_{2}=\frac{2 e^{*} L}{\pi u} \Gamma\left[1-\frac{2}{g}\right]\left\{\left[\left(\bar{E}_{J, 1}\right)^{2}+\left(\bar{E}_{J, 2}\right)^{2}\right] \sin \left[\frac{\pi}{g}\right]\right. \\
& \left.+\bar{E}_{J, 1} \bar{E}_{J, 2} \cos (\varphi) \cos \left[\frac{2 e^{*} V L}{v_{\mathrm{pl}}}+\frac{\pi}{g}\right]\right\}\left[\frac{4 L e^{*} V}{\pi u}\right]^{\frac{2}{g}-1}
\end{aligned}
$$

Eq.(146) contains an "interference" contribution (proportional to $\cos (\varphi)$ ), representing the cumulative effect of a bias voltage applied to junction 1 on the current flowing across junction 2, and of a bias voltage applied to junction 2 on the current flowing across junction 1 . This is a remarkable result of the application of the boundary sine Gordon techniques to SQUID devices. Treating the plasmon modes as modes of the dynamical fields, allows for an explicit computation of the interference contributions to the dc-current. 
The circulating current across the dc-SQUID, $J$, may be computed as the difference between the currents flowing across the two junctions, $I_{J}=I_{1}-I_{2}$. The result is

$$
\left.J=\frac{4 e^{*} \bar{E}_{J, 1} \bar{E}_{J, 2} L}{\pi v_{\mathrm{pl}}} \Gamma\left[1-\frac{2}{g}\right] \sin (\varphi) \sin \left[\frac{2 e^{*} V L}{v_{\mathrm{pl}}}+\frac{\pi}{g}\right]\right\}\left[\frac{4 L e^{*} V}{\pi v_{\mathrm{pl}}}\right]^{\frac{2}{g}-1}
$$

which, again, comes from pertinently taking into account correlation functions of the plasmon modes computed using the boundary sine Gordon theory.

\subsection{The dc-SQUID at the strongly coupled fixed point}

At the strongly coupled fixed point, as long as one neglects phase slips at the junctions, one should not expect any resistance across the junctions. As a consequence, the external voltage $V$ is added by biasing the left chiral mode of the plasmon field at each lead with respect to the right chiral mode, with opposite biases in the two contacts. As discussed in section (7.2) for the chain, this is accounted for by shifting the field $\Phi(x, t)$ according to

$$
\Phi(x, t) \rightarrow \Phi(x, t)-\frac{e^{*} V}{v_{\mathrm{pl}}} x
$$

Due to Eqs.(128, 129, 148), one has that, at finite $V$, also $\Phi_{u / d}(x, t)$ must be shifted as

$$
\Phi_{u / d}(x, t) \rightarrow \Phi_{u / d}(x, t)-\frac{e^{*} V}{2 v_{\mathrm{pl}}} x
$$

Eqs.(131) imply that

$$
X(x, t) \rightarrow X(x, t)-\frac{e^{*} V}{v_{\mathrm{pl}}} x \quad ; \quad \xi(x, t) \rightarrow \xi(x, t)
$$

which is equivalent to adding to the total Hamiltonian a voltage dependent contribution given by

$$
\tilde{H}_{V}=-\frac{e^{*} V g}{\pi} \int_{0}^{L} d x \frac{\partial X(x)}{\partial x}=-\frac{e^{*} V g}{2 \pi v_{\mathrm{pl}}} \int_{0}^{L} d x \frac{\partial \Theta_{X}(x)}{\partial \tau}
$$


The current operators across the two junctions are now written as

$$
j_{1 / 2}(i \tau)=-i \frac{e^{*}}{2 \pi}\left[\frac{\partial \Theta_{X}(i \tau)}{\partial \tau} \pm \frac{\partial \Theta_{\xi}(i \tau)}{\partial \tau}\right]=j_{X}(i \tau) \pm j_{\xi}(i \tau)
$$

An useful representation may be derived by adding to the total Euclidean action $S_{E}\left[\Theta_{X}, \Theta_{\xi}, a_{X}, a_{\xi}\right]$ the source term given by

$$
\delta S_{E}^{\text {Source }}=\frac{i e^{*}}{2 \pi L} \int_{0}^{\beta} d \tau \int_{0}^{L} d x\left[a_{X}(\tau) \frac{\partial \Theta_{X}(i \tau)}{\partial \tau}+a_{\xi}(\tau) \frac{\partial \Theta_{\xi}(i \tau)}{\partial \tau}\right]
$$

and by taking the functional derivatives of $S_{E}\left[\Theta_{X}, \Theta_{\xi}, a_{X}, a_{\xi}\right]$ with respect to $a_{X}, a_{\xi}$, after shifting the dual fields according to

$$
\Theta_{X}(x, i \tau) \rightarrow \Theta_{X}(x, i \tau)-2 i \frac{e^{*} g v_{\mathrm{pl}}}{L} A_{X}(\tau)-i g e^{*} V \tau
$$

and

$$
\Theta_{\xi}(x, i \tau) \rightarrow \Theta_{\xi}(x, i \tau)-2 i \frac{e^{*} g v_{\mathrm{pl}}}{L} A_{\xi}(\tau)+i \frac{\varphi}{2 L} \tau
$$

with $A_{X}^{\prime}(\tau)=a_{X}(\tau), A_{\xi}^{\prime}(\tau)=a_{\xi}(\tau)$

The Euclidean action in the dual representation is then given by

$$
\begin{aligned}
& \tilde{S}_{E}=\sum_{a=X, \xi} \frac{1}{4 \pi(2 g)} \int_{0}^{\beta} d \tau \int_{0}^{L} d x\left[\frac{1}{v_{\mathrm{pl}}}\left(\frac{\partial \Theta_{a}(x, i \tau)}{\partial \tau}\right)^{2}+v_{\mathrm{pl}}\left(\frac{\partial \Theta_{a}(x, i \tau)}{\partial x}\right)^{2}\right] \\
& -2 Y_{1} \int_{0}^{\beta} d \tau \cos \left[\frac{1}{2}\left(\Theta_{X}(i \tau)+\Theta_{\xi}(i \tau)\right)+i\left[g e^{*} V-v_{\mathrm{pl}} \frac{\varphi}{4 L}\right] \tau+i \frac{2 e^{*} g v_{\mathrm{pl}}}{L}\left[A_{\xi}(\tau)+A_{X}(\tau)\right]\right] \\
& -2 Y_{2} \int_{0}^{\beta} d \tau \cos \left[\frac{1}{2}\left(-\Theta_{X}(i \tau)+\Theta_{\xi}(i \tau)\right)-i\left[g e^{*} V+v_{\mathrm{pl}} \frac{\varphi}{4 L}\right] \tau+i \frac{2 e^{*} g v_{\mathrm{pl}}}{L}\left[A_{\xi}(\tau)-A_{X}(\tau)\right]\right] \\
& +\frac{1}{4 \pi(2 g)} \int_{0}^{\beta} d \tau \int_{0}^{L} d x\left\{\left[2 g e^{*} V+2 e^{*} g v_{\mathrm{pl}} \frac{a_{X}(\tau)}{L}\right]^{2}+\left[2 e^{*} g v_{\mathrm{pl}} \frac{a_{\xi}(\tau)}{L}+v_{\mathrm{pl}} \frac{\varphi}{4 L}\right]^{2}\right\} .(156)
\end{aligned}
$$


As a result of Eq.(156), one finds that the current operators $j_{X}, j_{\xi}$ may be written as

$$
\begin{aligned}
& j_{X}(i \tau)=\frac{g\left(e^{*}\right)^{2}}{\pi} V+4 i \frac{e^{*} g v_{\mathrm{pl}} Y_{1}}{L} \sin \left[\frac{1}{2}\left(\Theta_{X}(i \tau)+\Theta_{\xi}(i \tau)\right)+i\left[g e^{*} V+v_{\mathrm{pl}} \frac{\varphi}{4 L}\right] \tau\right] \\
& -2 i \frac{e^{*} g v_{\mathrm{pl}} Y_{2}}{L} \sin \left[\frac{1}{2}\left(-\Theta_{X}(i \tau)+\Theta_{\xi}(i \tau)\right)-i\left[g e^{*} V+v_{\mathrm{pl}} \frac{\varphi}{4 L}\right] \tau\right] \quad, \quad \text { (157) }
\end{aligned}
$$

and by

$$
\begin{aligned}
& j_{\xi}(i \tau)=\frac{e^{*} g v_{\mathrm{pl}}}{4 \pi L} \varphi+4 i \frac{e^{*} g v_{\mathrm{pl}} Y_{1}}{L} \sin \left[\frac{1}{2}\left(\Theta_{X}(i \tau)+\Theta_{\xi}(i \tau)\right)+i\left[g e^{*} V-v_{\mathrm{pl}} \frac{\varphi}{4 L}\right] \tau\right] \\
& +4 i \frac{e^{*} g u Y_{2}}{L} \sin \left[\frac{1}{2}\left(-\Theta_{X}(i \tau)+\Theta_{\xi}(i \tau)\right)-i\left[g e^{*} V+v_{\mathrm{pl}} \frac{\varphi}{4 L}\right] \tau\right]
\end{aligned}
$$

From Eqs.(157]158), one sees that, to the lowest order in the phase slip contribution $\left(Y_{1}=Y_{2}=0\right)$, the stationary component of the circulating current reduces to the value of the Josephson current at zero voltage, $\frac{e^{*} g u}{4 \pi L} \varphi$, while the conduction current is given by $\frac{g\left(e^{*}\right)^{2}}{\pi} V$, just as for a single chain at the strongly coupled fixed point.

Higher-order corrections, including the effects of phase slips, may be computed following the same steps used in the previous section. The result is

$$
\begin{aligned}
& \delta j_{X}=-\frac{4 e^{*} g\left(Y_{1}\right)^{2}}{\pi}\left\{\Gamma[1-2 g]\left[\frac{\Gamma\left[-\frac{a_{-}}{2}+g\right]}{\Gamma\left[1-\frac{a_{-}}{2}-g\right]}-\frac{\Gamma\left[\frac{a_{-}}{2}+g\right]}{\Gamma\left[1+\frac{a_{-}}{2}-g\right]}\right]\right\} \\
& +\frac{4 e^{*} g\left(Y_{2}\right)^{2}}{\pi}\left\{\Gamma[1-2 g]\left[\frac{\Gamma\left[\frac{a_{+}}{2}+g\right]}{\Gamma\left[1+\frac{a_{+}}{2}-g\right]}-\frac{\Gamma\left[-\frac{a_{+}}{2}+g\right]}{\Gamma\left[1-\frac{a_{+}}{2}-g\right]}\right]\right\},
\end{aligned}
$$

with

$$
a_{\mp}=\frac{2 g e^{*} V L}{\pi u} \mp \frac{u \varphi}{\pi}
$$

and by

$$
\delta j_{\xi}=-\frac{4 e^{*} g\left(Y_{1}\right)^{2}}{\pi}\left\{\Gamma[1-2 g]\left[\frac{\Gamma\left[-\frac{a_{-}}{2}+g\right]}{\Gamma\left[1-\frac{a_{-}}{2}-g\right]}-\frac{\Gamma\left[\frac{a_{-}}{2}+g\right]}{\Gamma\left[1+\frac{a_{-}}{2}-g\right]}\right]\right\}
$$




$$
-\frac{4 e^{*} g\left(Y_{2}\right)^{2}}{\pi}\left\{\Gamma[1-2 g]\left[\frac{\Gamma\left[\frac{a_{+}}{2}+g\right]}{\Gamma\left[1+\frac{a_{+}}{2}-g\right]}-\frac{\Gamma\left[-\frac{a_{+}}{2}+g\right]}{\Gamma\left[1-\frac{a_{+}}{2}-g\right]}\right]\right\},
$$

For $\varphi \sim 0$ and for $\left|a_{ \pm}\right| \ll 1$, one may expand Eqs. (159]161) to the first order in $a_{ \pm}$. In the symmetric case $\left(Y_{1}=Y_{2}\right)$, this provides a correction to the slope of both the dc-current and of the stationary component of the Josephson current, so that one gets

$$
\delta j_{X} \approx \frac{\left(e^{*}\right)^{2} g}{2 \pi}\left\{1+\frac{16 Y^{2} g L}{\pi u} C \Gamma[1-2 g]\right\} V
$$

and

$$
\delta j_{\xi} \approx \frac{e^{*} g v_{\mathrm{pl}}}{4 \pi L}\left\{1+\frac{16 Y^{2} g L}{\pi u} C \Gamma[1-2 g]\right\} \varphi
$$

with

$$
C=\frac{\partial}{\partial x}\left\{\frac{\Gamma[z+g]}{\Gamma[1-z+g]}-\frac{\Gamma[z+g]}{\Gamma[1+z-g]}\right\}_{z=0} .
$$

Eqs.(162,163) show in a rather simple context the way in which relevant perturbation may affect both the Josephson and the normal currents across the device.

\section{Comments and conclusions}

We provided a framework where two-boundary sine Gordon models may be used to investigate the relevant nonperturbative features, occurring in the renormalization of the Josephson couplings $E_{J, i}$ of a multi-junction SQUID. As pointed out in Ref.[7], this is needed since -for large fluctuations of the phase of the order parameter- the effective boundary conditions on the plasmon fields depend on the Josephson energy of the junction. Our analysis shows that, when $g>1$ and $L>L_{*}$, the Josephson couplings affect the dynamics of the plasmon field only as a boundary term, while, for $L<L_{*}$, they can be regarded as quantum impurities in a superconducting loop. For $g<1$, instead, the Josephson couplings have to be always regarded as quantum impurities. 
The boundary field theory approach to SQUID devices proposed in this paper turns out to be very powerful in providing a systematic procedure for computing not only the Josephson currents (and the leading corrections induced by the relevant perturbations), but also the dc-currents flowing in a SQUID device, due to externally applied voltages. For pertinent choices of the infrared cutoff, the results of the boundary field theory well reproduce what has been obtained in Ref. [9]7, allowing, in addition, to appreciate new and closer connections between the theories of Josephson superconducting devices, quantum brownian motion, fermionic quantum wires, and quantum impurity problems. Furthermore, for dc-SQUID devices, our analysis points out the existence of new remarkable interference effects between the currents flowing through each junction.

We investigated here SQUID-devices with one or two junctions. For these systems, we have found two stable regimes characterized by stable fixed points of the pertinent RG equations, driven by the strength of the bare Josephson energy. It would be interesting to ascertain if superconducting loops with more than two junctions may exhibit new (in addition to the by now well known weakly and strongly coupled fixed points) renormalization group fixed points at a finite Josephson coupling. Furthermore, it could be instructive to investigate the properties of a multi-junction SQUID device, for which $g=1$, since the boundary sine-Gordon model provides an exact solution for this value of the Luttinger parameter. We feel that multi-junction superconducting loops may become an interesting laboratory for testing the physical properties of field theories describing interacting Luttinger liquids with pertinent boundary interactions.

\section{Acknowledgments}

We thank Ian Affleck, Leonid Glazman, Hubert Saleur and Gordon W. Semenoff for inspiring discussions and useful correspondence at various stages of this project. We benefited from conversations with Maria Cristina Diamantini, Gianluca Grignani, Francesco P. Mancini, Arianna Montorsi, Mario Rasetti, Arturo Tagliacozzo and Andrea Trombettoni. This work has been partly supported by the MIUR National Project Josephson Networks for Quantum Coherence and Information (grant No.2004027555). 


\section{A Scaling properties of boundary interaction strengths}

In this appendix we shall derive the scaling laws for boundary interaction operators.

Let us consider a one-dimensional system described by the Euclidean action

$$
S=S_{0}-\sum_{\ell=1}^{M} \lambda_{\ell} \int_{0}^{\beta} d \tau \Phi_{\ell}(\tau)
$$

where $S_{0}$, the action for $K$ independent massless Klein-Gordon fields, $\left\{\phi_{j}\right\}$, with pertinent boundary conditions, is given by:

$$
S_{0}=\frac{g}{4 \pi} \sum_{j=1}^{K} \int_{0}^{\beta} d \tau \int_{0}^{L} d x\left[\frac{1}{v_{\mathrm{pl}}}\left(\frac{\partial \phi_{j}}{\partial \tau}\right)^{2}+v_{\mathrm{pl}}\left(\frac{\partial \phi_{j}}{\partial \tau}\right)^{2}\right] .
$$

The boundary operators $\left\{\Phi_{\ell}(\tau)\right\}$ are functionals of the fields $\left\{\phi_{j}\right\}$ with scaling dimension $h_{\ell}$. As a consequence, for $\tau \rightarrow \tau^{\prime}$, one gets

$$
G_{\ell}\left(\tau-\tau^{\prime}\right)=\left\langle\Phi_{\ell}(\tau) \Phi_{\ell^{\prime}}\left(\tau^{\prime}\right)\right\rangle_{0} \propto \delta_{\ell, \ell^{\prime}} \frac{1}{\left[2 \pi v_{\mathrm{pl}}\left(\tau-\tau^{\prime}\right) / L\right]^{2 h_{\ell}}}
$$

where $\langle\ldots\rangle_{0}$ denotes averaging with respect to the "free" action $S_{0}$, while $L$ is the "size" of the system.

It is well known that, in order to introduce scale invariant interaction terms, one needs to define dimensionless coupling constants, given by $g_{\ell}(a)=\lambda_{\ell} a^{1-h_{\ell}}$, where $\lambda_{\ell}$ is the coupling constant appearing in Eq. A.1. and $a$ is a short distance cutoff. To first order in the coupling strengths, the renormalization group equations are given by

$$
\frac{d g_{\ell}(a)}{d \ln \left(a / a_{0}\right)}=\left[1-h_{\ell}\right] g_{\ell}(a)
$$

Higher order corrections to the renormalization group equations come from nontrivial short-distance fusion rules of the $\Phi_{\ell}$ 's, which are represented by the O.P.E.'s

$$
\Phi_{\ell}(\tau) \Phi_{\ell^{\prime}}\left(\tau^{\prime}\right) \approx_{\tau^{\prime} \rightarrow \tau} \sum_{k} \frac{C_{\ell \ell^{\prime} \ell^{\prime \prime}}}{\left|\tau-\tau^{\prime}\right|^{h_{\ell}+h_{\ell}^{\prime}-h_{\ell} \prime}} \Phi_{\ell^{\prime \prime}}(\tau)
$$


If the O.P.E.'s coefficients $C_{\ell \ell^{\prime} \ell^{\prime}}$ are different than zero, the scaling equations get contributions which are of second order in the couplings. To derive them, one should start from the partition function

$$
Z=Z_{*}\left\langle\exp \left[\sum_{\ell=1}^{M} g_{\ell}(a) \int_{0}^{\beta} d \tau a^{-1+h_{\ell}} \Phi_{\ell}(\tau)\right]\right\rangle_{0}
$$

Upon introducing the cutoffs $a$ and $L$ and by expanding Eq.(A.6) up to the third-order in the running coupling strengths, one gets

$$
\begin{aligned}
& \frac{Z}{Z_{*}} \approx 1+\sum_{\ell, \ell^{\prime}=1}^{M} g_{\ell} g_{\ell^{\prime}} \int_{0}^{L / v_{\mathrm{pl}}} d \tau \int_{0}^{L / v_{\mathrm{pl}}} d \tau^{\prime} a^{-1+h_{\ell}} a^{-1+h_{\ell^{\prime}}} \theta\left(\tau-\tau^{\prime}-\frac{a}{v_{\mathrm{pl}}}\right)\left\langle\Phi_{\ell}(\tau) \Phi_{\ell^{\prime}}\left(\tau^{\prime}\right)\right\rangle_{0} \\
& +\sum_{\ell, \ell^{\prime}, \ell^{\prime \prime}=1}^{M} g_{\ell} g_{\ell^{\prime}} g_{\ell^{\prime \prime}} \int_{0}^{\frac{L}{u}} d \tau \int_{0}^{L / v_{\mathrm{pl}}} d \tau^{\prime} \int_{0}^{L / v_{\mathrm{pl}}} d \tau^{\prime \prime} a^{-1+h_{\ell}} a^{-1+h_{\ell^{\prime}}} a^{-1+h_{\ell^{\prime \prime}} \times} \\
& \theta\left(\tau-\tau^{\prime}-\frac{a}{v_{\mathrm{pl}}}\right) \theta\left(\tau^{\prime}-\tau^{\prime \prime}-\frac{a}{v_{\mathrm{pl}}}\right)\left\langle\Phi_{\ell}(\tau) \Phi_{\ell^{\prime}}\left(\tau^{\prime}\right) \Phi_{\ell^{\prime \prime}}\left(\tau^{\prime \prime}\right)\right\rangle_{0}
\end{aligned}
$$

If one rescales the cutoff, $a \rightarrow(1+\epsilon) a$, with $\epsilon \ll 1$, additional contributions to the coupling constant renormalization arise, as a result of configurations where the arguments of two fields lie between $a$ and $(1+\epsilon) a$. The ensuing renormalization is derived by means of the identity

$$
\begin{aligned}
& \theta\left(\tau-\tau^{\prime}-\frac{a}{v_{\mathrm{pl}}}(1+\epsilon)\right) \theta\left(\tau^{\prime}-\tau^{\prime \prime}-\frac{a}{v_{\mathrm{pl}}}(1+\epsilon)\right) \approx \theta\left(\tau-\tau^{\prime}-\frac{a}{v_{\mathrm{pl}}}\right) \theta\left(\tau^{\prime}-\tau^{\prime \prime}-\frac{a}{v_{\mathrm{pl}}}\right) \\
& -\epsilon \frac{a}{v_{\mathrm{pl}}}\left[\delta\left(\tau-\tau^{\prime}-\frac{a}{v_{\mathrm{pl}}}\right) \theta\left(\tau^{\prime}-\tau^{\prime \prime}-\frac{a}{v_{\mathrm{pl}}}\right)+\theta\left(\tau-\tau^{\prime}-\frac{a}{v_{\mathrm{pl}}}\right) \delta\left(\tau^{\prime}-\tau^{\prime \prime}-\frac{a}{v_{\mathrm{pl}}}\right)\right] .(\text { A. } 8)
\end{aligned}
$$

Thus, the third-order contribution to Eq. A.7 renomalizes the second-order term with

$$
\begin{aligned}
& 2 \epsilon \sum_{\ell^{\prime}, \ell^{\prime \prime}=1}^{M} g_{\ell^{\prime}} g_{\ell^{\prime \prime}} \sum_{\ell}^{M}\left[g_{\ell} C_{\ell, \ell^{\prime}, \ell^{\prime \prime}}\right] \int_{0}^{L / v_{\mathrm{pl}}} d \tau^{\prime} \int_{0}^{L / v_{\mathrm{pl}}} d \tau^{\prime \prime} \theta\left(\tau^{\prime}-\tau^{\prime \prime}-\frac{a}{v_{\mathrm{pl}}}\right) \times \\
& a^{-1+h_{\ell^{\prime}} a^{-1+h_{\ell^{\prime \prime}}} \Phi_{\ell^{\prime}}\left(\tau^{\prime}\right) \Phi_{\ell^{\prime \prime}}\left(\tau^{\prime \prime}\right) .} .
\end{aligned}
$$

By setting $\epsilon=\ln \left(a / a_{0}\right)$ in Eq.(A.6), it is straightforward, but tedious, to derive 
explicit expression for the nonlinear terms appearing in the RG equations for the running coupling strengths; the final result is

$$
\frac{d g_{\ell}(a)}{d \ln \left(a / a_{0}\right)}=\left[1-h_{\ell}\right] g_{\ell}(a)+\sum_{\ell^{\prime}, \ell^{\prime \prime}=1}^{M} C_{\ell, \ell^{\prime}, \ell^{\prime \prime}} g_{\ell^{\prime}}(a) g_{\ell^{\prime \prime}}(a)
$$

The RG equations in Eq. (A.10) may be rewritten as

$$
\frac{d g_{\ell}}{d \ln \left(a / a_{0}\right)}-\frac{\partial C[\{g\}]}{\partial g_{\ell}}=0
$$

where $C[\{g\}]$ is given by

$$
C[\{g\}]=\frac{1}{2} \sum_{\ell=1}^{N}\left[1-h_{\ell}\right] g_{\ell}^{2}+\frac{1}{3} \sum_{\ell, \ell^{\prime}, \ell^{\prime \prime}=1}^{M} C_{\ell, \ell^{\prime}, \ell^{\prime \prime}} g_{\ell} g_{\ell^{\prime}} g_{\ell^{\prime \prime}} .
$$

The RG fixed points coincide with the extrema of the function $C[\{g\}]$, that is, with the set of values of $\{g\},\left\{g^{*}\right\}$, such that

$$
\frac{\partial C\left[\left\{g^{*}\right\}\right]}{\partial g_{\ell}}=0 \quad, \forall \ell=1, \ldots, M
$$

Due to its properties, $C$ may also be identified with the "boundary entropy" of the system [32].

We would like to point out that the thermodynamics limit may be achieved either by sending either $a$ or $L$ to $\infty$. In fact, sending $a$ to $\infty$ amounts to cut off high momenta contributions, which amounts to send the size of the system to $\infty$. This implies that scaling may be realized either using $a / a_{0}$, or $L / L_{*}$ (as it has been done in this paper), as scaling parameters.

\section{B Boundary vertex operators}

As an example of correlation functions computed with both $\beta$ and $L$ finite, in this appendix we derive the correlators of the normal-ordered vertices $V_{X, \pm 1}(\tau)=: \exp [ \pm i X(i \tau)]:$, and $V_{\xi, \pm 1}(i \tau)=: \exp [ \pm i \xi(i \tau)]$ : introduced in section 3 , where $X(x, t)$ obeys Neumann boundary condions at both boundary $x=0$ and $x=L$, while $\xi(x, t)$ obeys Dirichlet boundary conditions at the inner boundary, Neumann at the outer boundary. 
The partition function $Z_{X}=\operatorname{Tr}\left[-\beta H_{X}\right]$, where $H_{X}$ is the Hamiltonian for the field $X$, has been derived in Eq.(84). The result is

$$
Z_{X}=\frac{1}{\eta(q)} \sum_{m \in Z} \exp \left[-\beta \frac{2 g \pi v_{\mathrm{pl}}}{L} m^{2}\right] \text {. }
$$

The two-vertex correlation function, $G_{X}\left(\tau_{1}, \tau_{2}\right)$, is then given by

$$
G_{X}\left(\tau_{1}, \tau_{2}\right)=\frac{1}{Z_{X}} \operatorname{Tr}\left[T_{\tau}\left\{: e^{-i X\left(i \tau_{1}\right)}:: e^{i X\left(i \tau_{2}\right)}:\right\} e^{-\beta H_{X}}\right]
$$

As it happens for the partition function, also $G_{X}$ is factorized into a contribution from the oscillatory modes, times a contribution from the zero modes. The trace over the oscillatory modes yields

$$
\mathbf{T}_{\mathrm{osc}}=\prod_{n=1}^{\infty} \mathbf{T}_{n}
$$

where

$$
\begin{aligned}
& \mathbf{T}_{n}=\operatorname{Tr} \mathbf{T}_{\tau}\left\{\exp \left[i \sqrt{\frac{1}{g}} \frac{a\left(-k_{n}\right)}{n} e^{v_{\mathrm{pl}} k_{n} \tau_{1}}\right] \exp \left[-i \sqrt{\frac{1}{g}} \frac{a\left(k_{n}\right)}{n} e^{-v_{\mathrm{pl}} k_{n} \tau_{1}}\right] \times\right. \\
& \exp \left[i \sqrt{\frac{2}{g}} \frac{a_{X}\left(-k_{n}\right)}{n} e^{v_{\mathrm{p} 1} k_{n} \tau_{2}}\right] \exp \left[i \sqrt{\frac{2}{g}} \frac{a_{X}\left(k_{n}\right)}{n} e^{-v_{\mathrm{p} 1} k_{n} \tau_{2}}\right] q^{a_{X}\left(-k_{n}\right) a_{X}\left(k_{n}\right)}
\end{aligned}
$$

The trace in Eq.(B.4) may be computed by resorting to a coherent state decomposition, which uses the basis of coherent states $\left|x_{n}\right\rangle$, defined by

$$
a_{X}\left(k_{n}\right)\left|x_{n}\right\rangle=\sqrt{n} x_{n}\left|x_{n}\right\rangle \Rightarrow\left|x_{n}\right\rangle=e^{-\frac{\left|\vec{\xi}_{n}\right|^{2}}{2}} e^{\frac{x_{n} a_{X}\left(-k_{n}\right)}{n}}|0\rangle
$$

and the decomposition of the identity given by

$$
\mathbf{I}=\left(\frac{1}{\pi}\right) \int d x_{n} e^{-\frac{\left(x_{n}\right)^{2}}{2}}\left|x_{n}\right\rangle\left\langle x_{n}\right|
$$

The derivation is tedious, but straightforward [37]. The final result is

$$
\mathbf{T}_{n}=\left[\frac{1}{1-q^{n}}\right]^{2} \exp \left[-\frac{4}{g n}\left(\frac{q^{n}}{1-q^{n}}\right)\right] \times
$$




$$
\exp \left\{\left(\frac{2}{g\left(1-q^{n}\right)}\right) \frac{e^{-v_{\mathrm{pl}} k_{n}\left|\tau_{1}-\tau_{2}\right|}}{n}+\left(\frac{2 q^{n}}{g\left(1-q^{n}\right)}\right) \frac{e^{v_{\mathrm{pl}} k_{n}\left|\tau_{1}-\tau_{2}\right|}}{n}\right\} .
$$

As a consequence, one gets

$$
\begin{aligned}
& \mathbf{T}_{\mathrm{osc}}=\prod_{n=1}^{\infty} \mathbf{T}_{n}=\frac{1}{\eta(q)} \exp \left[-\frac{4}{g} \sum_{n=1}^{\infty} \frac{q^{n}}{n\left(1-q^{n}\right)}\right] \times \\
& \exp \left[\frac{1}{g} \sum_{n=1}^{\infty} \frac{\left(e^{-k_{n} v_{\mathrm{p} 1}\left|\tau_{1}-\tau_{2}\right|}\right)^{n}}{\left(1-q^{n}\right)}+\frac{1}{g} \sum_{n=1}^{\infty} \frac{q^{n}\left(e^{-k_{n} v_{\mathrm{p} 1}\left|\tau_{1}-\tau_{2}\right|}\right)^{n}}{\left(1-q^{n}\right)}\right] .
\end{aligned}
$$

Using the Jacobi's triple product identity [37], one finally obtains

$$
\prod_{n=1}^{\infty} \mathbf{T}_{n}=\frac{1}{\eta(q)}\left\{\frac{\theta_{1}\left(\frac{i v_{\mathrm{pl}}\left(\tau_{1}-\tau_{2}\right)}{2 L} \mid i \frac{v_{\mathrm{pl}} \beta}{L}\right)}{\theta_{1}^{\prime}\left(0 \mid i \frac{v_{\mathrm{pl}} \beta}{L}\right)}\right\}^{-\frac{1}{g}}
$$

where $\theta_{1}(\zeta \mid \tau)$ is Jacobi's $\theta_{1}$ elliptic function, defined as

$$
\theta_{1}(\zeta \mid \tau)=-i \sum_{n \in Z}(-1)^{n} e^{\pi \tau\left(n+\frac{1}{2}\right)^{2}} e^{2 i \pi \zeta\left(n+\frac{1}{2}\right)}
$$

By tracing over the zero mode and applying Poisson's resummation formula, one eventually gets

$$
G_{X}\left(\tau_{1}, \tau_{2}\right)=\theta_{3}\left(\frac{\tau u}{2 g L} \mid i \frac{\beta u}{2 g L}\right)\left[\frac{\theta_{1}\left(i v_{\mathrm{pl}} \frac{\left(\tau_{1}-\tau_{2}\right)}{2 L} \mid i \frac{v_{\mathrm{pl}} \beta}{L}\right)}{\theta_{1}^{\prime}\left(0 \mid i \frac{v_{\mathrm{pl}} \beta}{2 L}\right)}\right]^{-\frac{2}{g}} .
$$

where the $\theta_{3}$-function is defined as

$$
\theta_{3}(\zeta \mid \tau)=\sum_{n \in Z} e^{i \pi \tau \frac{n^{2}}{2}} e^{2 \pi n \zeta}
$$

By performing similar computations, one obtains

$$
\begin{aligned}
& G_{\xi}\left(\tau_{1}, \tau_{2}\right)=\frac{1}{Z_{X}} \operatorname{Tr}\left[T_{\tau}\left\{: e^{-i \xi\left(i \tau_{1}\right)}:: e^{i \xi\left(i \tau_{2}\right)}:\right\} e^{-\beta H_{\xi}}\right]= \\
& \theta_{3}\left(\frac{\tau u}{2 g L} \mid i \frac{\beta u}{2 g L}\right)\left[\frac{\theta_{4}\left(i v_{\mathrm{pl}} \frac{\left(\tau_{1}-\tau_{2}\right)}{2 L} \mid i \frac{v_{\mathrm{pl}} \beta}{L}\right)}{\theta_{1}^{\prime}\left(0 \mid i \frac{v_{\mathrm{pl}} \beta}{2 L}\right)}\right]^{-\frac{2}{g}} .
\end{aligned}
$$


where the $\theta_{4}$-function is defined as

$$
\theta_{4}(\zeta \mid \tau)=\sum_{n \in Z}(-1)^{n} e^{i \pi \tau \frac{n^{2}}{2}} e^{2 \pi i n \zeta}
$$

When taking the limit $\beta \rightarrow \infty$, the correlators in Eqs. (B.11B.13) give the ones that we have used in the paper.

\section{References}

[1] For a classical review, see M.H. Devoret, D. Esteve, C. Urbina, J. Martinis, A. Cleland and J. Clarke, in Quantum Tunnelling in Condensed Media, Yu. Kagan and A. J. Leggett eds., Elsevier, Amsterdam, (1992).

[2] G. Schoen and A. D. Zaikin, Phys. Rep. 198,(1990), 237.

[3] A. O. Caldeira and A. J. Leggett, Ann. Phys. (N.Y) 149,(1983), 374.

[4] M. H. Devoret, D. Esteve, H. Grabert, G.L. Ingold, H. Pothier, C.Urbina, Phys. Rev. Lett. 64, (1990), 1824; S. M. Girvin, L. Glazman, M. Jonson, D.R. Penn, M.D. Stiles, Phys. Rev.Lett. 64,(1990), 3183.

[5] H. Grabert and U. Weiss, Phys. Rev. Lett. 54,(1985), 1605; M. P. A. Fisher and A. T. Dorsey, Phys. Rev. Lett. 54,(1985),1609; A. T. Dorsey, M.P.A. Fisher and M. S. Wartak, Phys. Rev.A33, (1986), 1117.

[6] D. V. Averin, Yu. A. Nazarov, A. A. Odintsov, Physica B165-166,(1990), 945; G. Falci, V. Bubanja, G. Schoen Europhys. Lett. 16,(1991), 109 and Z. Phys. B85,(1991), 451; G. L. Ingold, H. Grabert and U. Eberhardt, Phys. Rev. B50,(1994), 395.

[7] F. W. J. Hekking and L. I. Glazman, Phys. Rev. B 55, (1997), 6551.

[8] J. E. Mooij and G. Schoen Phys. Rev. Lett. 55, (1985), 114; O. Buisson, P. Xavier and J. Richard Phys. Rev. Lett. 73, (1994), 3153.

[9] M. P. A. Fisher and W. Zwerger, Phys. Rev. B32, (1985), 6190; C. L. Kane and M. P. A. Fisher, Phys. Rev. Lett., 68,(1992), 1220 and Phys. Rev. B46,(1992), 15233.

[10] A. I. Vainshtein, V. I. Zakharov, V. A. Novikov and M. A. Shifman, Sov. Phys. Usp. 25,(1982), 195; P. W. Anderson and G. Yuval, Phys. Rev. Lett., 23,(1969), 89; D. R. Hamann, Phys. Rev. Lett. 23, (1969), 95; G. Yuval and P.W. Anderson, Phys. Rev. B1, (1970), 1522; P. W. Anderson, G. Yuval and D. R. Hamann Phys. Rev. B1, (1970), 4464.

[11] Y. Makhlin, G. Schoen and A. Shnirman, Rev. Mod. Phys. 73, (2001), 357. 
[12] J. E. Mooij, T. P. Orlando, L. Levitov, L. Tian, C. H. van der Wal and S. Lloyd, Science 285, (1999), 1036; M. V. Feigel'man, V. B. Geshkenbein, L. B. Ioffe and G. Blatter, J. Low. Temp. Phys. 118, (2000), 805; L. B. Ioffe, V. B. Geshkenbein, M. V. Feigel'man, A. L. Fauchere, G. Blatter, Nature 398, (1999), 679; C. H. van der Wal, A. C. J. ter Haar, F. K. Wilhelm, R. N. Schouten, C. J. P. M. Harmans, T. P. Orlando, S. Lloyd and J. E. Mooij, Science 290, (2000), 773; G. Blatter, V. B. Gesjkenbein and L. b. Ioffe. Rev. B63, (2001), 174511.

[13] T. P. Orlando,J. E. Mooij, L. Tian, C. H. van der Wal, L. S. Levitov, S. Lloyd, J. J. Mazo, Phys. Rev. B60, (1999), 15398.

[14] R. F. Voss and R. A. Webb, Phys. Rev. Lett. 47, (1981), 265; nJ. M. Martinis, M. H. Devoret and J. Clarke, Phys. Rev. B35, (1987), 4682; J. Clarke, A. N. Cleland, M. H. Devoret, D. Esteve and J. M. Martinis, Science 239, (1988), 992; R. Rouse, S. Han and J. E. Lukens, Phys. Rev. Lett. 75, (1995), 1614; P. Silvestrini, V. G. Palmieri, B. Ruggiero and M. Russo, Phys. Rev. Lett. 79, (1997), 3046; J. R. Friedman, V. Patel, W. Chen, S. K. Tolpygo and J.E. Lukens, Nature 406, (2000), 43.

[15] A. Le Clair, G. Mussardo, H. Saleur, S. Shorik, Nucl. Phys. B453 (1995) 581; J.-,S. Caux, H. Saleur and F. Siano, Nucl. Phys. B 672, (2003), 411 and Phys. Rev. Lett. 88 (2002) 106402.

[16] J. Cardy, hep-th/0411189, to appear as entry in Encyclopedia of Mathematical Physics.

[17] J. M. Luttinger, J. Math. Phys. 4, (1963), 1154; S. Tomonaga, Prog. Theor. Phys. 5, (1950), 544.

[18] H. J. Shulz, G. Cuniberti and P. Pieri in Field Theories for Low-Dimensional Correlated Systems, G. Morandi, P. Sodano, V. Tognetti and A. Tagliacozzo eds., Springer, Berlin.

[19] C. Chamon, M. Oshikawa and I. Affleck, Phys. Rev. Lett. 91, (2003), 206403; Journal of Statistical Mechanics JSTAT/2006/P02008.

[20] K.A. Matveev and L.I. Glazman, Physica B189, (1993) 266.

[21] D. Giuliano and P. Sodano, Nucl. Phys. B 711, (2005), 480.

[22] I. Affleck, Nucl Phys. B336,(1990), 517; I. Affleck and A. W. W. Ludwig, Nucl. Phys. B352, (1991), 849; Nucl.Phys. B360, (1991), 341; Phys. Rev. Lett. 67, (1991) 161; Phys. Rev. B48, (1993), 7292.

[23] C. L. Kane, M. P. A. Fisher, Phys. Rev. B46,(1992), 1220.

[24] P. Fendley, A.W.W. Ludwig, H. Saleur, Phys. Rev. Lett. 74, (1995) 3005; Phys. Rev. B52,(1995), 8934; C. L. Kane and M. P. A. Fisher, Phys. Rev. B46,(1992), 15233.

[25] I. Affleck, M. Oshikawa and H. Saleur, Nucl. Phys. B594, (2001), 535; H. Yi and C. L. Kane, cond-mat 9602099. 
[26] I. Affleck, J. S. Caux and A. M. Zagoskin, Phys. Rev. B62, (2000), 1433.

[27] F. D. M. Haldane, Phys. Rev. Lett. 45, (1980) 1358; J. L. Black, V. J. Emery: Phys. Rev. B 23, (1981) 429; M. P. M. denNijs: Phys. Rev. B 23, (1981) 6111. P. Gueret, N. Blanc, R.Germann, and H. Rothuizen, Phys. Rev. Lett. 68, (1992) 1896.

[28] K. Bardakci and A. Konechny, hep-th/0009214; S. A. Harvey, D. Kutasov, and E. J. Martinec, hep-th/0003101.

[29] A. Sen, J. High Energy Phys. 027, (1999) 9912.

[30] A. LeClair, M. E. Peskin, and C. R. Preitschopf, Nucl. Phys. B317, (1989) 411; J. A. Harvey, D. Kutasov, E. G. Martinec, and G. Moore, hep-th/0111154.

[31] E. Gava, K. S. Narain, and N. H. Sarmadi, Nucl. Phys. B504, (1997) 214; A. Sen J. High Energy Phys. 09, (1998), 013; Int. Jour. Mod. Phys. A14, (1999), 4061.

[32] I. Affleck and A. A. Ludwig, Phys. Rev. Lett. 67, (1991), 161.

[33] S. Coleman, Phys. Rev. D 11, (1975) 2088; D. J. Amit, Y. Y. Goldshmidt and G. Grinstein, J. Phys. A 13, (1980) 585.

[34] K. A. Matveev, A. I. Larkin and L. I. Glazman, Phys. Rev. Lett. 89,(2002), 096802-1.

[35] L. I. Glazman and A. I. Larkin, Phys. Rev. Lett. 79, 3736-3739 (1997).

[36] M. Abramowitz, Handbook of Mathematical Functions, (United States. National Bureau of Standards. Applied mathematics series, 55, 1964).

[37] See, for example, M. B. Green, J. H. Schwartz and E. Witten, "Superstring Theory", vol. 2, Cambridge University Press Eds. 